\title{
United Arab Emirates: Statistical Appendix
}

This Statistical Appendix paper for the United Arab Emirates was prepared by a staff team of the International Monetary Fund as background documentation for the periodic consultation with the member country. It is based on the information available at the time it was completed on May 13, 2004. The views expressed in this document are those of the staff team and do not necessarily reflect the views of the government of the United Arab Emirates or the Executive Board of the IMF.

The policy of publication of staff reports and other documents by the IMF allows for the deletion of market-sensitive information.

To assist the IMF in evaluating the publication policy, reader comments are invited and may be sent by e-mail to publicationpolicy@imf.org.

Copies of this report are available to the public from

International Monetary Fund • Publication Services

700 19th Street, N.W. • Washington, D.C. 20431

Telephone: (202) 6237430 • Telefax: (202) 6237201

E-mail: publications@imf.org • Internet: http://www.imf.org

Price: $\$ 15.00$ a copy

\section{International Monetary Fund} Washington, D.C. 



\title{
INTERNATIONAL MONETARY FUND
}

\section{UNITED ARAB EMIRATES}

\author{
Statistical Appendix \\ Prepared by a staff team consisting of Mohamad Elhage, Van Can Thai, John Wilson, \\ Hamid Davoodi, and Holger Floerkemeier (all MCD) \\ Approved by the Middle East and Central Asia Department
}

May 13, 2004

Contents

Page

Statistical Appendix Tables

1. Selected Economic Indicators, 1999-2004 ..................................................................

2. Sectoral Origin of GDP at Constant 1995 Prices, 1998-2003 ……...................................

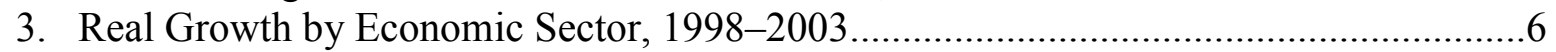

4. Sectoral Origin of GDP at Current Prices, 1998-2003 ……….....................................

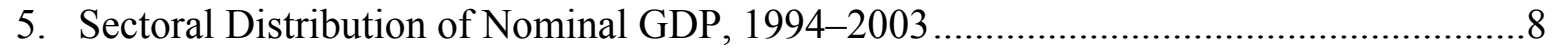

6. Use of Resources at Current Prices, 1998-2003 ......................................................... 9

7. Per Capital GDP and Distribution of GDP at Factor Cost by Emirate, 1998-2003 .......10

8. Gross Fixed Capital Formation by Sector at Current Prices, 1998-2003 .......................11

9. Oil and Gas Production, Exports, and Prices, 1998-2003 ……..................................12

10. NGLs, LNG, and Refined Product Exports, 1998-2003 ...........................................13

11. Average Crude Oil Prices, 1998-2003 ....................................................................14

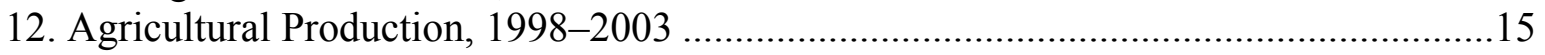

13. Population by Emirate, 1998-2003 .......................................................................

14. Sectoral Distribution of Civilian Employment, 1998-2003 …………...........................17

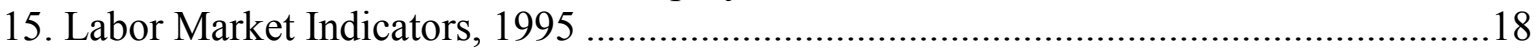

16. Average Annual Compensation by Economic Sector, 1998-2003 ................................19

17. Selected Price Indices, 1998-2003 ………………….............................................20

18. Consumer Price Index by Major Components, 1999-2003 …………….......................21

19. Consolidated Government Finances, 1999-2003 ……………………………….......22

20. Government Current Expenditures by Economic Category and Emirate, 1999-2003 ...24

21. Federal Government Financial Operations, 1999-2003 ……………………….........25

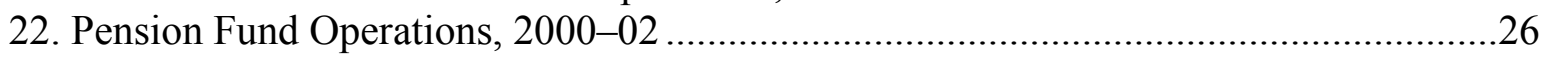

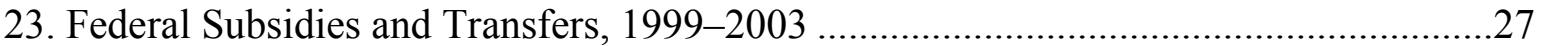

24. Federal Development Expenditures, 1999-2003 ………........................................28

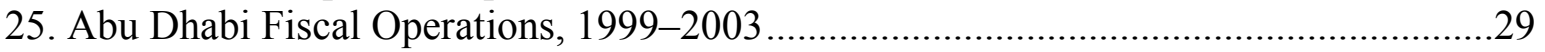

26. Abu Dhabi Development Expenditures, 1999-2003 …………………………............30

27. Abu Dhabi Government Transfers and Subsidies, 1999-2003 …………………….......31

28. Dubai Government Operations, 1999-2003 ………………………………….......32

29. Sharjah Government Fiscal Operations, 1999-2003 ..................................................33

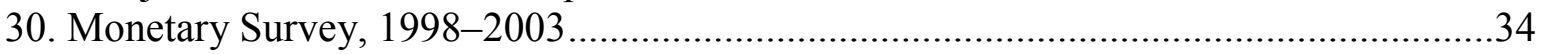

31. Factors Affecting Domestic Liquidity, 1998-2003 .......................................................35 
32. Summary Accounts of the Central Bank, 1998-2003 ..............................................36

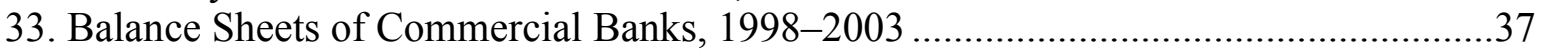

34. Balance Sheet of Restricted License Bank, 1998-2003 .............................................38

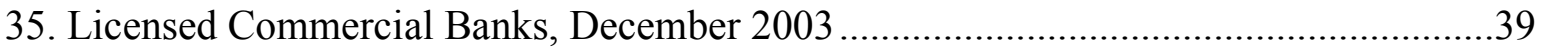

36. Balance of Payments, 1998-2003 (in U.S. dollars) ..................................................40

37. Balance of Payments, 1998-2003 (in U.A.E. dirhams) ..............................................42

38. Merchandise Imports by Harmonized System Sections, 1998-2002 .........................44

39. Merchandise Exports by Harmonized System Sections, 1999-2002 ...........................45

40. Direction of Trade: Imports, 1998-2002 .................................................................46

41. Direction of Trade: Exports, 1998-2003 ..............................................................47 
Table 1. United Arab Emirates: Selected Economic Indicators, 1999-2004

\begin{tabular}{|c|c|c|c|c|c|c|}
\hline & 1999 & 2000 & 2001 & 2002 & $\frac{\text { Prel. }}{2003}$ & $\frac{\text { Proj. }}{2004}$ \\
\hline & \multicolumn{6}{|c|}{ (In billions of U.A.E. dirhams) } \\
\hline \multirow[t]{2}{*}{ GDP at market prices } & 202.7 & 258.0 & 255.4 & 263.4 & 295.4 & 310.8 \\
\hline & \multicolumn{6}{|c|}{ (In millions of barrels per day) } \\
\hline \multicolumn{7}{|l|}{ Oil production and exports } \\
\hline Crude oil production $1 /$ & 2.26 & 2.41 & 2.44 & 2.27 & 2.59 & 2.47 \\
\hline Of which: Condensates & 0.18 & 0.22 & 0.32 & 0.33 & 0.33 & 0.33 \\
\hline Average U.A.E. crude price (U.S. dollars per barrel) & 17.93 & 27.51 & 23.91 & 24.75 & 28.11 & 29.50 \\
\hline & \multicolumn{6}{|c|}{ (In billions of cubic meters per year) } \\
\hline \multicolumn{7}{|l|}{ Natural gas production and exports } \\
\hline Natural gas production & 34.6 & 35.9 & 45.0 & 46.0 & 46.0 & 49.2 \\
\hline LNG exports & 7.2 & 7.1 & 7.5 & 7.3 & 7.3 & 7.6 \\
\hline NGL exports & 11.1 & 12.3 & 11.5 & 11.3 & 12.7 & 13.6 \\
\hline & \multicolumn{6}{|c|}{ (Annual percentage changes) } \\
\hline \multicolumn{7}{|l|}{ National income and prices } \\
\hline Real GDP (at factor cost) & 4.4 & 12.3 & 3.5 & 1.9 & 7.0 & 2.4 \\
\hline Crude oil, condensates, and natural gas & -4.5 & 13.1 & 1.7 & -8.1 & 13.8 & -5.3 \\
\hline Nonhydrocarbon sectors $2 /$ & 7.5 & 12.0 & 4.0 & 5.0 & 5.2 & 4.7 \\
\hline GDP deflator & 9.0 & 13.9 & -4.8 & 0.9 & 4.8 & 2.8 \\
\hline Consumer prices & 2.1 & 1.4 & 2.8 & 3.1 & 2.8 & 2.6 \\
\hline & \multicolumn{6}{|c|}{ (In percent of GDP) } \\
\hline \multicolumn{7}{|l|}{ Investment and saving } \\
\hline Gross domestic investment & 27.8 & 23.3 & 24.7 & 24.1 & 22.4 & 23.9 \\
\hline Government & 6.8 & 4.4 & 5.2 & 4.7 & 5.2 & 5.1 \\
\hline Nongovernment & 21.0 & 18.9 & 19.4 & 19.3 & 17.2 & 18.8 \\
\hline National saving & 29.5 & 40.6 & 34.1 & 29.0 & 30.9 & 31.3 \\
\hline Government & -1.6 & 7.8 & 0.7 & -2.4 & 2.7 & 2.5 \\
\hline Nongovernment & 31.1 & 32.7 & 33.4 & 31.4 & 28.1 & 28.8 \\
\hline \multicolumn{7}{|l|}{ Consolidated government finances } \\
\hline Revenue & 27.0 & 44.5 & 37.1 & 43.7 & 44.4 & 38.0 \\
\hline Hydrocarbon & 14.9 & 33.0 & 26.6 & 34.1 & 35.8 & 29.8 \\
\hline Nonhydrocarbon & 12.2 & 11.5 & 10.5 & 9.6 & 8.6 & 8.3 \\
\hline Of which: Investment income & 4.8 & 5.8 & 4.5 & 3.4 & 2.8 & 2.6 \\
\hline Expenditure & 37.3 & 32.0 & 37.4 & 32.9 & 30.7 & 29.9 \\
\hline \multicolumn{7}{|l|}{ Of which } \\
\hline Current & 28.6 & 26.9 & 30.0 & 27.5 & 25.2 & 24.6 \\
\hline Development & 8.0 & 4.6 & 7.0 & 5.0 & 5.1 & 5.0 \\
\hline Overall balance & -10.2 & 12.5 & -0.4 & 10.8 & 13.7 & 8.1 \\
\hline Overall balance (excluding investment income) & -15.0 & 6.6 & -4.9 & 7.4 & 10.9 & 5.5 \\
\hline (Excluding hydrocarbon revenue) & -29.9 & -26.3 & -31.5 & -26.7 & -24.9 & -24.3 \\
\hline Financing & 10.2 & -12.5 & 0.4 & -10.8 & -13.7 & -8.1 \\
\hline Domestic banks & 0.5 & -4.1 & -0.2 & -3.2 & -0.8 & -1.3 \\
\hline Other & 9.7 & -8.4 & 0.6 & -7.6 & -13.0 & -6.8 \\
\hline Government debt 3/ & 7.2 & 4.6 & 4.0 & 5.5 & 7.3 & 7.0 \\
\hline
\end{tabular}


Table 1. United Arab Emirates: Selected Economic Indicators, 1999-2004

\begin{tabular}{|c|c|c|c|c|c|c|}
\hline & 1999 & 2000 & 2001 & 2002 & $\frac{\text { Prel. }}{2003}$ & $\frac{\text { Proj. }}{2004}$ \\
\hline & \multicolumn{6}{|c|}{ (Changes in percent of initial stock of M2) } \\
\hline \multicolumn{7}{|l|}{ Money and credit } \\
\hline Broad money (M2) & 11.4 & 15.1 & 15.5 & 15.6 & 16.1 & 9.8 \\
\hline Foreign assets (net) & 3.2 & 20.2 & 8.7 & 20.1 & 1.9 & 1.7 \\
\hline Domestic assets & 8.3 & -5.1 & 6.7 & -4.5 & 14.2 & 7.9 \\
\hline \multicolumn{7}{|l|}{ Of which: } \\
\hline Claims on government (net) & 1.1 & -9.6 & -0.4 & -5.8 & -1.3 & -2.1 \\
\hline Claims on public sector enterprises & 0.3 & 0.2 & -0.4 & 1.3 & 3.5 & 0.2 \\
\hline \multirow[t]{2}{*}{ Claims on private sector } & 7.6 & 9.0 & 8.5 & 10.4 & 11.9 & 11.4 \\
\hline & \multicolumn{6}{|c|}{ (In percent per annum, end of period) } \\
\hline \multicolumn{7}{|l|}{ Average interest rates } \\
\hline Dirham (three-month interbank) & 5.53 & 6.53 & 3.50 & 1.79 & 1.28 & 1.17 \\
\hline Lending rate & 10.59 & 9.60 & 7.82 & 6.82 & 5.81 & 4.92 \\
\hline & \multicolumn{6}{|c|}{ (In billions of U.S. dollars) } \\
\hline \multicolumn{7}{|l|}{ External Sector } \\
\hline Exports & 36.5 & 49.6 & 47.5 & 51.2 & 60.8 & 63.6 \\
\hline Crude oil & 13.6 & 21.7 & 17.6 & 16.7 & 22.1 & 22.2 \\
\hline Petroleum products & 0.6 & 1.7 & 2.1 & 3.0 & 3.5 & 4.0 \\
\hline Gas & 2.3 & 3.7 & 3.3 & 3.1 & 3.9 & 4.2 \\
\hline Non-hydrocarbon exports & 6.3 & 7.5 & 8.5 & 10.6 & 11.3 & 13.1 \\
\hline Re-exports & 13.7 & 15.0 & 16.0 & 17.8 & 20.0 & 20.1 \\
\hline Imports, f.o.b. & -27.9 & -30.8 & -33.5 & -36.7 & -41.7 & -43.8 \\
\hline Trade balance & 8.6 & 18.8 & 14.0 & 14.5 & 19.1 & 19.8 \\
\hline Services (net) & -5.9 & -6.4 & -6.2 & -7.3 & -7.5 & -7.9 \\
\hline Income (net) & 2.2 & 3.8 & 2.9 & 0.8 & -0.1 & 0.5 \\
\hline Transfers (net) & -3.9 & -4.0 & -4.2 & -4.4 & -4.7 & -6.1 \\
\hline Private & -3.6 & -3.7 & -3.9 & -4.1 & -4.4 & -5.7 \\
\hline Official & -0.3 & -0.3 & -0.3 & -0.3 & -0.3 & -0.4 \\
\hline Current account balance & 0.9 & 12.2 & 6.5 & 3.5 & 6.9 & 6.3 \\
\hline (In percent of GDP) & 1.6 & 17.3 & 9.4 & 4.9 & 8.5 & 7.5 \\
\hline Overall balance & 1.5 & 2.8 & 0.5 & 1.1 & -0.2 & 0.6 \\
\hline Central Bank reserves & 10.9 & 13.8 & 14.3 & 15.3 & 15.1 & 15.7 \\
\hline In months of imports & 4.3 & 4.9 & 4.7 & 4.4 & 4.1 & 4.1 \\
\hline As percent of reserve money & 199.8 & 250.2 & 242.8 & 223.5 & 181.8 & 180.2 \\
\hline As percent of short-term debt $4 /$ & 191.9 & 168.6 & 162.4 & 164.6 & 163.4 & 185.6 \\
\hline Total external debt $5 /$ & 18.8 & 18.2 & 19.4 & 16.7 & 16.6 & 15.3 \\
\hline (In percent of GDP) & 34.0 & 26.0 & 27.9 & 23.3 & 20.7 & 18.0 \\
\hline \multicolumn{7}{|l|}{ Memorandum item: } \\
\hline Dirhams/U.S. dollar (end of period) & 3.6725 & 3.6725 & 3.6725 & 3.6725 & 3.6725 & 3.6725 \\
\hline
\end{tabular}

Sources: U.A.E. authorities; Bank for International Settlements (BIS); Organization for Economic Cooperation and Development (OECD); and IMF staff estimates.

1/ Crude oil output includes condensates, which are not subject to OPEC quotas.

2/ Includes refined products and liquid gas.

3/ Due to domestic banks; no official external debt is reported.

4/ Debt due within one year, from BIS/OECD statistics.

5/ Central Bank and commercial bank foreign liabilities, plus private non-banks (BIS source). 
Table 2. United Arab Emirates: Sectoral Origin of GDP at Constant 1995 Prices, 1998-2003

(In millions of U.A.E. dirhams)

\begin{tabular}{|c|c|c|c|c|c|c|}
\hline & 1998 & 1999 & 2000 & 2001 & 2002 & $\frac{\text { Prel. }}{2003}$ \\
\hline Gross domestic product & 182,913 & 190,884 & 214,327 & 221,751 & 226,011 & 241,828 \\
\hline Crude oil production & 47,517 & 45,360 & 51,310 & 52,181 & 47,956 & 54,578 \\
\hline Other sectors & 135,396 & 145,524 & 163,017 & 169,570 & 178,055 & 187,250 \\
\hline Agriculture & 6,231 & 7,346 & 8,733 & 8,500 & 8,738 & 8,956 \\
\hline Industry & 43,680 & 46,297 & 54,439 & 55,272 & 57,412 & 61,751 \\
\hline Mining and quarrying & 612 & 637 & 662 & 672 & 697 & 733 \\
\hline Manufacturing $1 /$ & 23,311 & 25,390 & 33,120 & 33,362 & 34,630 & 38,010 \\
\hline Electricity and water & 4,011 & 4,244 & 4,487 & 4,748 & 5,115 & 5,347 \\
\hline Construction & 15,746 & 16,026 & 16,170 & 16,490 & 16,970 & 17,661 \\
\hline Services & 85,485 & 91,881 & 99,845 & 105,798 & 111,905 & 116,543 \\
\hline Trade & 23,553 & 24,485 & 25,321 & 26,136 & 27,992 & 29,262 \\
\hline Wholesale and retail trade & 19,732 & 20,032 & 20,676 & 21,146 & 22,500 & 23,495 \\
\hline Restaurants and hotels & 3,821 & 4,453 & 4,645 & 4,990 & 5,492 & 5,767 \\
\hline \multicolumn{7}{|l|}{ Transportation, storage, and } \\
\hline Finance and insurance & 12,546 & 13,201 & 14,207 & 16,244 & 16,875 & 17,808 \\
\hline Real estate & 17,772 & 18,454 & 18,618 & 18,906 & 19,420 & 20,004 \\
\hline Government services & 19,296 & 21,430 & 24,296 & 25,547 & 26,895 & 27,280 \\
\hline Other services & 4,617 & 4,892 & 5,242 & 5,717 & 6,073 & 6,270 \\
\hline \multicolumn{7}{|l|}{ Less: } \\
\hline Imputed bank service charges & 5,195 & 4,758 & 4,050 & 5,065 & 5,481 & 5,773 \\
\hline
\end{tabular}

Source: Ministry of Planning.

1/ Includes natural gas and petroleum processing industries. 
Table 3. United Arab Emirates: Real Growth by Economic Sector, 1998-2003

(Change in percent)

\begin{tabular}{|c|c|c|c|c|c|c|}
\hline & 1998 & 1999 & 2000 & 2001 & 2002 & $\frac{\text { Prel. }}{2003}$ \\
\hline Gross domestic product & 1.4 & 4.4 & 12.3 & 3.5 & 1.9 & 7.0 \\
\hline Crude oil production & -7.6 & -4.5 & 13.1 & 1.7 & -8.1 & 13.8 \\
\hline Other sectors & 5.0 & 7.5 & 12.0 & 4.0 & 5.0 & 5.2 \\
\hline Agriculture & 4.2 & 17.9 & 18.9 & -2.7 & 2.8 & 2.5 \\
\hline Industry & 4.1 & 6.0 & 17.6 & 1.5 & 3.9 & 7.6 \\
\hline Mining and quarrying & 3.9 & 4.1 & 3.9 & 1.5 & 3.7 & 5.2 \\
\hline Manufacturing 1/ & 4.9 & 8.9 & 30.4 & 0.7 & 3.8 & 9.8 \\
\hline Electricity and water & 3.2 & 5.8 & 5.7 & 5.8 & 7.7 & 4.5 \\
\hline Construction & 3.3 & 1.8 & 0.9 & 2.0 & 2.9 & 4.1 \\
\hline Services & 5.6 & 7.5 & 8.7 & 6.0 & 5.8 & 4.1 \\
\hline Trade & 4.1 & 4.0 & 3.4 & 3.2 & 7.1 & 4.5 \\
\hline Wholesale and retail trade & 2.7 & 1.5 & 3.2 & 2.3 & 6.4 & 4.4 \\
\hline Restaurants and hotels & 11.5 & 16.5 & 4.3 & 7.4 & 10.1 & 5.0 \\
\hline \multicolumn{6}{|l|}{ Transportation, storage, } & 7.8 \\
\hline Finance and insurance & 16.0 & 5.2 & 7.6 & 14.3 & 3.9 & 5.5 \\
\hline Real estate & 3.4 & 3.8 & 0.9 & 1.5 & 2.7 & 3.0 \\
\hline Government services & 6.6 & 11.1 & 13.4 & 5.1 & 5.3 & 1.4 \\
\hline Other services & 4.2 & 6.0 & 7.2 & 9.1 & 6.2 & 3.2 \\
\hline \multicolumn{7}{|l|}{ Less: } \\
\hline Imputed bank service charges & 32.9 & -8.4 & -14.9 & 25.1 & 8.2 & 5.3 \\
\hline
\end{tabular}

Source: Ministry of Planning.

1/ Includes natural gas and petroleum processing industries. 
Table 4. United Arab Emirates: Sectoral Origin of GDP at Current Prices, 1998-2003

(In millions of U.A.E. dirhams)

\begin{tabular}{lrrrrrr}
\hline & & & & & & \\
& & & & & & \\
& 1998 & 1999 & 2000 & 2001 & 2002 & 2003 \\
& & & & & & \\
\hline & & & & & & \\
Gross domestic product & 177,360 & 201,797 & 257,979 & 254,236 & 261,370 & 293,121 \\
& & & & & & \\
Crude oil production & 37,402 & 49,794 & 86,690 & 74,990 & 72,552 & 93,369 \\
Other sectors & 139,958 & 152,003 & 171,289 & 179,246 & 188,818 & 199,752 \\
Agriculture & 6,325 & 7,551 & 9,047 & 8,862 & 9,105 & 9,359 \\
Industry & 45,125 & 48,232 & 56,916 & 58,165 & 60,660 & 65,169 \\
$\quad$ Mining and quarrying & 619 & 656 & 682 & 697 & 725 & 765 \\
$\quad$ Manufacturing 1/ & 23,987 & 26,539 & 34,762 & 35,132 & 36,673 & 40,100 \\
$\quad$ Electricity and water & 4,127 & 4,416 & 4,615 & 4,890 & 5,274 & 5,513 \\
$\quad$ Construction & 16,392 & 16,621 & 16,857 & 17,446 & 17,988 & 18,791 \\
Services & 88,508 & 96,220 & 105,326 & 112,219 & 119,053 & 125,224 \\
$\quad$ Trade & 25,005 & 26,255 & 27,294 & 28,273 & 30,386 & 32,119 \\
$\quad$ Wholesale and retail trade & 20,955 & 21,535 & 22,268 & 22,838 & 24,361 & 25,774 \\
$\quad$ Restaurants and hotels & 4,050 & 4,720 & 5,026 & 5,435 & 6,025 & 6,345 \\
$\quad$ Transportation, storage, and & & & & & & \\
$\quad$ communication & 13,347 & 15,247 & 17,247 & 19,595 & 21,742 & 23,629 \\
$\quad$ Finance and insurance & 13,023 & 13,663 & 14,862 & 16,845 & 17,314 & 18,394 \\
$\quad$ Real estate & 17,664 & 18,384 & 19,068 & 19,662 & 20,388 & 21,205 \\
$\quad$ Government services & 20,010 & 22,458 & 25,561 & 27,029 & 28,525 & 29,272 \\
$\quad$ Other services & 4,758 & 5,071 & 5,465 & 6,007 & 6,398 & 6,644 \\
$\quad$ Less: & & & & & & \\
$\quad$ Imputed bank service charges & 5,299 & 4,858 & 4,171 & 5,192 & 5,700 & 6,039 \\
& & & & & & \\
\hline
\end{tabular}

Source: Ministry of Planning.

1/ Includes natural gas and petroleum processing industries. 


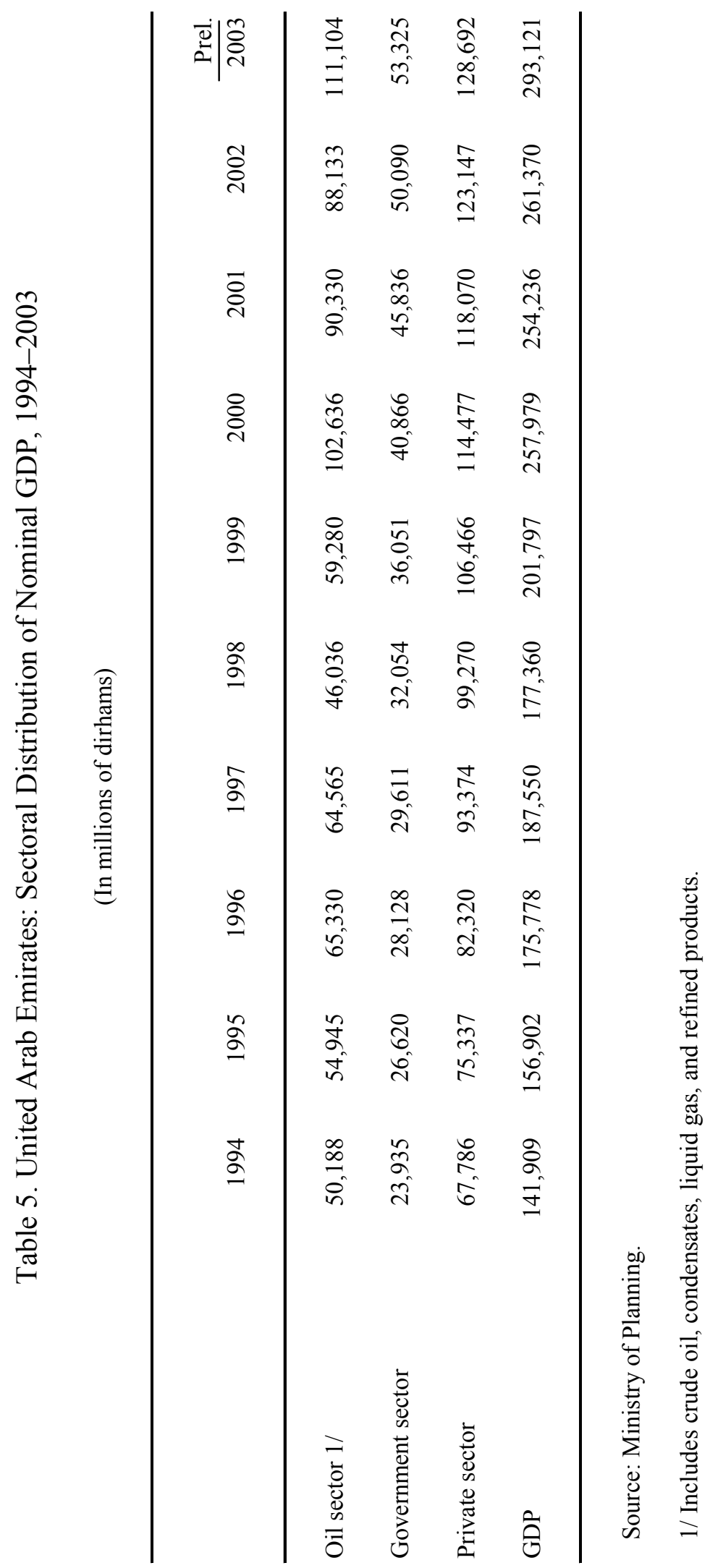


Table 6. United Arab Emirates: Use of Resources at Current Prices, 1998-2003

(In millions of U.A.E. dirhams)

\begin{tabular}{|c|c|c|c|c|c|c|}
\hline & 1998 & 1999 & 2000 & 2001 & 2002 & $\frac{\text { Prel. }}{2003}$ \\
\hline Consumption & 128,253 & 136,482 & 152,559 & 163,563 & 173,666 & 186,762 \\
\hline Public & 35,226 & 36,117 & 39,885 & 42,000 & 42,540 & 43,489 \\
\hline Private & 93,027 & 100,365 & 112,674 & 121,563 & 131,126 & 143,273 \\
\hline Fixed investment & 52,195 & 53,916 & 57,398 & 60,178 & 60,552 & 63,070 \\
\hline Public & 32,402 & 31,564 & 32,643 & 35,167 & 34,927 & 35,970 \\
\hline Private & 19,793 & 22,352 & 24,755 & 25,011 & 25,625 & 27,100 \\
\hline Change in inventories & 2,320 & 2,500 & 2,680 & 2,792 & 2,870 & 2,950 \\
\hline Domestic expenditure & 182,768 & 192,898 & 212,637 & 226,533 & 237,088 & 252,782 \\
\hline Net exports of goods and services & $-4,650$ & 9,800 & 46,610 & 28,875 & 26,271 & 42,579 \\
\hline Exports & 129,600 & 141,710 & 190,070 & 183,316 & 197,058 & 233,680 \\
\hline Imports & 134,250 & 131,910 & 143,460 & 154,441 & 170,787 & 191,101 \\
\hline GDP at market prices & 178,118 & 202,698 & 257,991 & 255,408 & 263,359 & 295,361 \\
\hline Plus: subsidies, less indirect taxes & -758 & -901 & $-1,268$ & $-1,172$ & $-1,989$ & $-2,240$ \\
\hline Subsidies & 1,814 & 1,780 & 1,794 & 1,813 & 1,277 & 1,282 \\
\hline Indirect taxes & 2,572 & 2,681 & 3,062 & 2,985 & 3,266 & 3,522 \\
\hline Equals: GDP at factor cost & 177,360 & 201,797 & 257,979 & 254,236 & 261,370 & 293,121 \\
\hline
\end{tabular}

Source: Ministry of Planning. 
Table 7. United Arab Emirates: Per Capita GDP and Distribution of GDP at Factor Cost by Emirate, 1998-2003

\begin{tabular}{|c|c|c|c|c|c|c|}
\hline Emirate & 1998 & 1999 & 2000 & 2001 & 2002 & $\frac{\text { Prel. }}{2003}$ \\
\hline & \multicolumn{6}{|c|}{ (In millions of U.A.E. dirhams) } \\
\hline Gross domestic product (GDP) & 177,360 & 201,797 & 257,979 & 254,236 & 261,370 & 293,121 \\
\hline Abu Dhabi & 96,772 & 112,194 & 159,624 & 148,439 & 151,016 & 173,957 \\
\hline Dubai & 49,876 & 55,810 & 62,335 & 66,028 & 69,244 & 74,869 \\
\hline Sharjah & 17,885 & 19,866 & 21,380 & 24,054 & 24,593 & 26,535 \\
\hline Ajman & 3,545 & 3,888 & 4,006 & 4,230 & 4,425 & 4,797 \\
\hline Umm al-Qaiwan & 1,197 & 1,306 & 1,405 & 1,439 & 1,504 & 1,647 \\
\hline Ras al-Khaimah & 5,218 & 5,636 & 5,940 & 6,318 & 6,636 & 7,159 \\
\hline \multirow[t]{2}{*}{ Fujairah } & 2,867 & 3,097 & 3,289 & 3,728 & 3,952 & 4,156 \\
\hline & \multicolumn{6}{|c|}{ (In U.A.E. dirhams) } \\
\hline Per capita GDP & 62,583 & 66,534 & 79,451 & 72,889 & 69,624 & 72,537 \\
\hline Abu Dhabi & 87,656 & 94,999 & 126,085 & 108,986 & 102,732 & 109,338 \\
\hline Dubai & 60,676 & 63,062 & 65,478 & 64,167 & 62,269 & 62,184 \\
\hline Sharjah & 38,298 & 39,892 & 40,416 & 42,801 & 41,058 & 41,722 \\
\hline Ajman & 23,477 & 23,564 & 22,380 & 21,582 & 20,580 & 20,412 \\
\hline Umm al-Qaiwan & 28,500 & 29,022 & 28,673 & 27,673 & 25,496 & 26,573 \\
\hline Ras al-Khaimah & 32,818 & 34,158 & 34,535 & 34,906 & 35,489 & 36,711 \\
\hline Fujairah & 32,213 & 32,947 & 32,890 & 35,170 & 35,282 & 35,217 \\
\hline
\end{tabular}

Source: Ministry of Planning. 
Table 8. United Arab Emirates: Gross Fixed Capital Formation by Sector at Current Prices, 1998-2003

(In millions of U.A.E. dirhams)

\begin{tabular}{|c|c|c|c|c|c|c|}
\hline & 1998 & 1999 & 2000 & 2001 & 2002 & $\frac{\text { Prel. }}{2003}$ \\
\hline Total fixed investment & 52,195 & 53,916 & 57,398 & 60,178 & 60,552 & 63,070 \\
\hline Oil sector & 6,385 & 7,180 & 7,280 & 7,530 & 7,925 & 8,200 \\
\hline Other sectors & 45,810 & 46,736 & 50,118 & 52,648 & 52,627 & 54,870 \\
\hline Agriculture & 1,554 & 1,413 & 1,534 & 1,997 & 2,097 & 1,716 \\
\hline Industry & 14,108 & 14,497 & 17,374 & 18,149 & 18,708 & 19,948 \\
\hline Mining and quarrying & 120 & 123 & 154 & 164 & 186 & 206 \\
\hline Manufacturing 1/ & 6,793 & 7,740 & 9,570 & 9,583 & 10,387 & 11,133 \\
\hline Electricity and water & 4,470 & 3,764 & 4,600 & 5,359 & 5,067 & 5,327 \\
\hline Construction & 2,725 & 2,870 & 3,050 & 3,043 & 3,068 & 3,282 \\
\hline Services & 30,148 & 30,826 & 31,210 & 32,502 & 31,822 & 33,206 \\
\hline Trade & 4,320 & 4,717 & 5,492 & 5,744 & 6,326 & 6,795 \\
\hline Wholesale and retail trade & 2,039 & 2,267 & 2,505 & 2,517 & 2,734 & 3,061 \\
\hline Restaurants and hotels & 2,281 & 2,450 & 2,987 & 3,227 & 3,592 & 3,734 \\
\hline $\begin{array}{l}\text { Transportation, storage, and } \\
\text { communications }\end{array}$ & 10,310 & 10,852 & 9,722 & 10,760 & 10,465 & 11,202 \\
\hline Finance and insurance & 236 & 279 & 289 & 603 & 606 & 355 \\
\hline Real estate & 9,617 & 10,478 & 11,096 & 9,849 & 10,031 & 10,272 \\
\hline Other services $2 /$ & 5,665 & 4,500 & 4,611 & 5,546 & 4,394 & 4,582 \\
\hline
\end{tabular}

Source: Ministry of Planning.

1/ Includes investment in natural gas and petroleum-processing industries.

2/ Includes repair services. 
Table 9. United Arab Emirates: Oil and Gas Production, Exports, and Prices, 1998-2003

\begin{tabular}{|c|c|c|c|c|c|c|}
\hline & 1998 & 1999 & 2000 & 2001 & 2002 & $\frac{\text { Prel. }}{2003}$ \\
\hline & \multicolumn{6}{|c|}{ (In millions of barrels per day) } \\
\hline Crude oil and condensates & 2.43 & 2.26 & 2.41 & 2.44 & 2.27 & 2.59 \\
\hline Crude oil & 2.26 & 2.08 & 2.19 & 2.12 & 1.94 & 2.26 \\
\hline Abu Dhabi & 2.02 & 1.85 & 1.99 & 1.94 & 1.77 & 2.10 \\
\hline Dubai and others 1/ & 0.24 & 0.23 & 0.20 & 0.18 & 0.17 & 0.16 \\
\hline Condensates & 0.17 & 0.18 & 0.22 & 0.32 & 0.33 & 0.33 \\
\hline Refinery output & 0.23 & 0.23 & 0.30 & 0.52 & 0.56 & 0.56 \\
\hline Oil and product exports & 2.28 & 2.17 & 2.33 & 2.28 & 2.18 & 2.50 \\
\hline Crude oil & 2.03 & 1.90 & 2.05 & 1.94 & 1.85 & 2.17 \\
\hline Abu Dhabi & 1.83 & 1.70 & 1.85 & 1.80 & 1.61 & 1.93 \\
\hline Dubai and others $1 /$ & 0.20 & 0.20 & 0.20 & 0.14 & 0.14 & 0.14 \\
\hline Condensates & 0.17 & 0.18 & 0.13 & 0.10 & 0.10 & 0.10 \\
\hline \multirow[t]{2}{*}{ Refined products } & 0.08 & 0.09 & 0.15 & 0.24 & 0.33 & 0.33 \\
\hline & \multicolumn{6}{|c|}{ (In billions of cubic meters) } \\
\hline Natural gas production 2/ & 33.40 & 34.60 & 35.90 & 45.00 & 46.00 & 46.00 \\
\hline LNG exports & 7.41 & 7.22 & 7.11 & 7.46 & 7.34 & 7.34 \\
\hline NGL exports & 11.87 & 11.11 & 12.30 & 11.52 & 11.28 & 12.75 \\
\hline \multirow[t]{2}{*}{ Natural gas domestic consumption } & 30.40 & 31.40 & 33.40 & 38.10 & 39.30 & 40.00 \\
\hline & \multicolumn{6}{|c|}{ (In millions of U.S. dollars) } \\
\hline Oil and product exports & 10,400 & 14,200 & 23,400 & 19,733 & 19,727 & 25,653 \\
\hline Crude oil & 9,200 & 12,400 & 20,400 & 17,612 & 16,679 & 22,115 \\
\hline Abu Dhabi & 8,200 & 11,000 & 18,500 & 15,642 & 14,518 & 19,801 \\
\hline Dubai and others & 1,000 & 1,400 & 1,900 & 1,166 & 1,216 & 1,369 \\
\hline Condensates & 800 & 1,200 & 1,300 & 804 & 945 & 945 \\
\hline Refined products & 400 & 600 & 1,700 & 2,121 & 3,048 & 3,538 \\
\hline $\begin{array}{l}\text { LNG, NGL, LPG exports } \\
\text { of which: }\end{array}$ & 2,112 & 2,289 & 3,668 & 3,305 & 3,099 & 3,912 \\
\hline LNG & 926 & 782 & 1,156 & 1,280 & 1,172 & 1,307 \\
\hline NGL & 1,186 & 1,507 & 2,512 & 2,025 & 1,927 & 2,605 \\
\hline Total oil and gas exports & 12,512 & 16,489 & 27,068 & 23,038 & 22,826 & 29,565 \\
\hline \multicolumn{7}{|l|}{ Natural gas domestic consumption } \\
\hline \multicolumn{7}{|l|}{ Memorandum item: } \\
\hline $\begin{array}{l}\text { Average oil export price } \\
\text { (in U.S. dollars per barrel) }\end{array}$ & 12.50 & 17.93 & 27.51 & 23.91 & 24.75 & 28.11 \\
\hline $\begin{array}{l}\text { Average Abu Dhabi oil export price } \\
\text { (in U.S. dollars per barrel) }\end{array}$ & 12.34 & 17.70 & 27.36 & 23.81 & 24.64 & 28.11 \\
\hline
\end{tabular}

Source: Abu Dhabi National Oil Company.

1/ Sharjah and Ras Al-Khaimah.

2/ Net after re-injection into wells. 
Table 10. United Arab Emirates: NGLs, LNG, and Refined Product Exports, 1998-2003 (In thousands of metric tons)

\begin{tabular}{|c|c|c|c|c|c|c|}
\hline & 1998 & 1999 & 2000 & 2001 & 2002 & $\begin{array}{c}\text { Prel. } \\
2003\end{array}$ \\
\hline Total NGL exports & 8,601 & 7,950 & 8,916 & 8,349 & 8,175 & 9,237 \\
\hline LPG & 6,291 & 6,002 & 6,451 & 6,000 & 5,900 & 6,791 \\
\hline Abu Dhabi & 5,541 & 5,252 & 5,701 & 5,200 & 5,100 & 6,091 \\
\hline Dubai & 300 & 300 & 300 & 300 & 300 & 275 \\
\hline Sharjah & 450 & 450 & 450 & 500 & 500 & 425 \\
\hline Pentane plus & 2,310 & 1,948 & 2,465 & 2,349 & 2,275 & 2,446 \\
\hline Abu Dhabi & 1,810 & 1,548 & 1,915 & 1,799 & 1,725 & 1,946 \\
\hline \multicolumn{7}{|l|}{ Of which } \\
\hline ADNOC & 833 & 685 & 946 & 920 & 900 & 1,091 \\
\hline ADGAS & 477 & 500 & 530 & 479 & 425 & 480 \\
\hline Other emirates & 500 & 400 & 550 & 550 & 550 & 500 \\
\hline LNG exports (ADGAS) & 5,372 & 5,229 & 5,149 & 5,406 & 5,320 & 5,320 \\
\hline Refined products exports & 3,688 & 4,031 & 6,789 & 10,095 & 11,948 & 11,625 \\
\hline
\end{tabular}

Source: Abu Dhabi National Oil Company. 
Table 11. United Arab Emirates: Average Crude Oil Prices, 1998-2003

(In U.S. dollars per barrel)

\begin{tabular}{lcccccc}
\hline & 1998 & 1999 & 2000 & 2001 & 2002 & 2003 \\
& & & & & & \\
& & & & & & \\
Murban & 12.61 & 17.97 & 27.73 & 24.15 & 24.88 & 28.37 \\
Lower Zakum & 12.61 & 17.99 & 27.78 & 24.19 & 24.90 & 28.39 \\
Umm-Shaif & 12.37 & 17.74 & 27.53 & 23.91 & 24.68 & 28.14 \\
Upper Zakum & 11.78 & 17.10 & 26.40 & 22.98 & 24.12 & 27.53 \\
Dubai platts & 12.17 & 17.21 & 26.15 & 22.81 & 23.81 & 26.79 \\
& & & & & & \\
\hline
\end{tabular}

Source: Abu Dhabi National Oil Company. 
Table 12. United Arab Emirates: Agricultural Production, 1998-2003

(In thousands of metric tons)

\begin{tabular}{lrrrrrr}
\hline & & & & & & Prel. \\
& 1998 & 1999 & 2000 & 2001 & 2002 & 2003 \\
& & & & & & \\
\hline & & & & & & \\
Plant products & & & & & & \\
$\quad$ Dates and fruits & 333 & 577 & 798 & 795 & 800 & 822 \\
Vegetables & 1,554 & 1,722 & 2,622 & 579 & 467 & 453 \\
Other crops & 507 & 584 & 1,495 & 3,176 & 4,308 & 4,436 \\
& & & & & & \\
Animal products & 22 & 23 & 25 & 26 & 28 & 29 \\
Meat & 26 & 29 & 27 & 28 & 29 & 30 \\
$\quad$ Poultry & 252 & 237 & 266 & 310 & 322 & 340 \\
Eggs (in millions) & 118 & 142 & 163 & 179 & 202 & 215 \\
Milk and dairy products & & & & & & \\
& 119 & 115 & 117 & 124 & 141 & 148 \\
Fish products & & & & & & \\
\hline
\end{tabular}

Sources: Ministry of Planning and Ministry of Agriculture and Fisheries. 
Table 13. United Arab Emirates: Population by Emirate, 1998-2003

(In thousands)

\begin{tabular}{lrrrrrr}
\hline Emirate & 1998 & 1999 & 2000 & 2001 & 2002 & $\frac{\text { Prel. }}{2003}$ \\
\hline Abu Dhabi & 1,104 & 1,181 & 1,266 & 1,362 & 1,470 & 1,591 \\
Dubai & 822 & 885 & 952 & 1,029 & 1,112 & 1,204 \\
Sharjah & 467 & 498 & 529 & 562 & 599 & 636 \\
Ajman & 151 & 165 & 179 & 196 & 215 & 235 \\
Umm al-Qaiwan & 42 & 45 & 49 & 52 & 59 & 62 \\
Ras al-Khaimah & 159 & 165 & 172 & 181 & 187 & 195 \\
Fujairah & 89 & 94 & 100 & 106 & 112 & 118 \\
$\quad$ Total & 2,834 & 3,033 & 3,247 & 3,488 & 3,754 & 4,041 \\
\hline
\end{tabular}

Source: Ministry of Planning. 
Table 14. United Arab Emirates: Sectoral Distribution of Civilian Employment, 1998-2003 1/

(In thousands)

\begin{tabular}{|c|c|c|c|c|c|c|}
\hline & 1998 & 1999 & 2000 & 2001 & 2002 & $\frac{\text { Prel. }}{2003}$ \\
\hline Civilian employment & 1,437 & 1,632 & 1,738 & 1,929 & 2,087 & 2,189 \\
\hline Oil sector & 21 & 22 & 23 & 26 & 27 & 28 \\
\hline Other sectors & 1,417 & 1,610 & 1,714 & 1,903 & 2,060 & 2,161 \\
\hline Agriculture & 113 & 120 & 120 & 154 & 163 & 168 \\
\hline Industry & 482 & 513 & 547 & 588 & 654 & 698 \\
\hline Mining and quarrying & 4 & 4 & 4 & 4 & 5 & 5 \\
\hline Manufacturing 2/ & 197 & 210 & 226 & 247 & 273 & 298 \\
\hline Electricity, gas, and water & 24 & 28 & 30 & 31 & 32 & 33 \\
\hline Construction & 257 & 271 & 287 & 305 & 344 & 362 \\
\hline Services & 822 & 981 & 1,047 & 1,161 & 1,243 & 1,295 \\
\hline Trade & 300 & 392 & 409 & 455 & 497 & 525 \\
\hline Wholesale and retail trade & 236 & 322 & 337 & 371 & 406 & 429 \\
\hline Restaurants and hotels & 63 & 70 & 72 & 84 & 91 & 96 \\
\hline Transport and communications & 100 & 105 & 108 & 127 & 131 & 134 \\
\hline Finance and insurance & 23 & 23 & 23 & 25 & 26 & 26 \\
\hline Real estate & 35 & 37 & 42 & 56 & 61 & 63 \\
\hline Government services & 155 & 182 & 204 & 214 & 237 & 249 \\
\hline Social and personal services & 66 & 71 & 81 & 91 & 93 & 98 \\
\hline Domestic household services & 144 & 170 & 180 & 193 & 198 & 200 \\
\hline
\end{tabular}

Source: Ministry of Planning.

1/ Excludes defense personnel.

2/ Includes natural gas and petroleum-processing industries. 


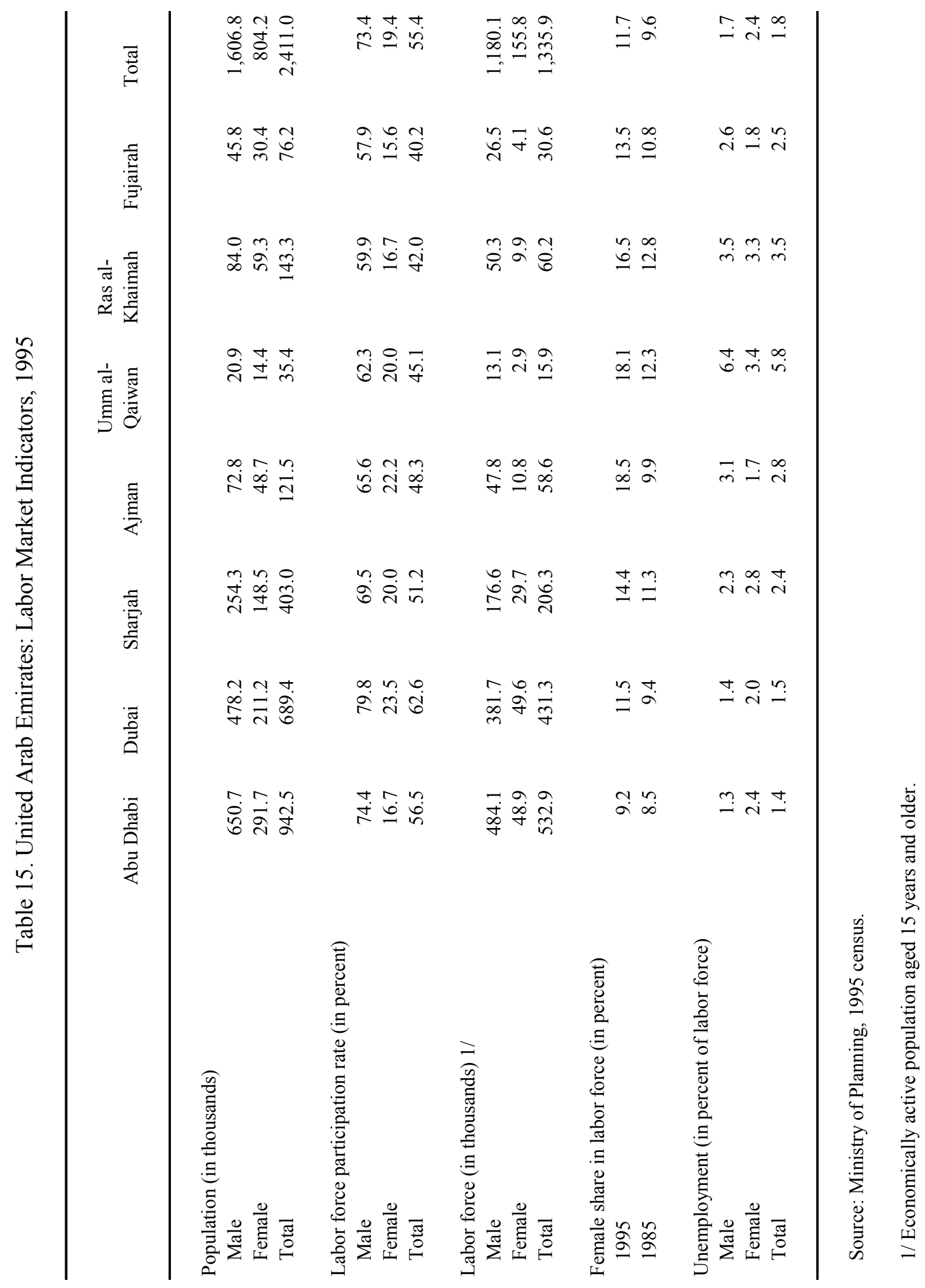


Table 16. United Arab Emirates: Average Annual Compensation by Economic Sector, 1998-2003 1/2/

(In thousands of U.A.E. dirhams)

\begin{tabular}{|c|c|c|c|c|c|c|}
\hline & 1998 & 1999 & 2000 & 2001 & 2002 & $\frac{\text { Prel. }}{2003}$ \\
\hline Crude oil & 88.2 & 84.7 & 86.6 & 85.6 & 85.2 & 85.7 \\
\hline Agriculture & 16.0 & 15.7 & 15.8 & 16.4 & 15.6 & 15.5 \\
\hline Mining and quarrying & 27.0 & 26.1 & 26.8 & 27.0 & 28.6 & 27.7 \\
\hline Manufacturing 3/ & 21.3 & 20.9 & 23.0 & 22.7 & 22.0 & 22.0 \\
\hline Electricity, gas, and water & 47.0 & 41.3 & 42.2 & 42.5 & 42.8 & 43.0 \\
\hline Construction & 38.2 & 37.7 & 32.5 & 31.6 & 29.8 & 29.7 \\
\hline Wholesale and retail trade 4/ & 27.3 & 20.8 & 26.1 & 24.9 & 25.4 & 25.9 \\
\hline Restaurants and hotels & 18.5 & 17.5 & 22.2 & 22.7 & 22.2 & 22.2 \\
\hline Transport, storage, and communications & 40.7 & 40.1 & 56.0 & 52.9 & 59.2 & 59.9 \\
\hline Finance and insurance & 93.8 & 95.0 & 102.5 & 116.1 & 117.2 & 119.9 \\
\hline Real estate & 27.9 & 27.3 & 27.2 & 23.1 & 22.3 & 22.3 \\
\hline Government services & 79.4 & 70.2 & 84.5 & 86.1 & 83.9 & 82.7 \\
\hline Social and personal services & 28.6 & 27.5 & 27.1 & 25.9 & 25.9 & 25.6 \\
\hline Household services & 10.1 & 9.0 & 9.1 & 10.1 & 10.3 & 10.4 \\
\hline Weighted average & 37.5 & 34.3 & 37.7 & 36.8 & 36.5 & 36.4 \\
\hline
\end{tabular}

Source: Ministry of Planning.

1/ Calculated on the basis of wages and allowances and the number of workers in each sector.

2/ Excludes defense personnel.

$3 /$ Includes natural gas and petroleum processing industries.

4/ Includes repair services. 
Table 17. United Arab Emirates: Selected Price Indices, 1998-2003

(Annual averages, $1995=100)$

\begin{tabular}{|c|c|c|c|c|c|c|}
\hline & 1998 & 1999 & 2000 & 2001 & 2002 & $\frac{\text { Prel. }}{2003}$ \\
\hline GDP deflator & 97.0 & 105.7 & 120.4 & 114.7 & 115.4 & 120.2 \\
\hline Crude oil deflator & 78.7 & 109.8 & 169.0 & 143.7 & 150.4 & 170.8 \\
\hline Non-oil deflator & 103.4 & 104.5 & 105.1 & 105.7 & 105.9 & 106.5 \\
\hline Consumer price index & 106.9 & 109.2 & 110.7 & 113.8 & 117.2 & 120.3 \\
\hline
\end{tabular}

Source: Ministry of Planning. 
Table 18. United Arab Emirates: Consumer Price Index by Major Components, 1999-2003 (Annual averages, $1995=100)$

\begin{tabular}{|c|c|c|c|c|c|c|}
\hline & Weights $1 /$ & 1999 & 2000 & 2001 & 2002 & $\frac{\text { Prel. }}{2003}$ \\
\hline Consumer price index & 100.00 & 109.2 & 110.7 & 113.8 & 117.2 & 120.3 \\
\hline Foodstuffs, beverages, and tobacco & 14.43 & 112.8 & 113.4 & 114.5 & 115.1 & 116.8 \\
\hline Ready-made clothes and footwear & 6.74 & 113.5 & 114.2 & 115.5 & 116.2 & 118.5 \\
\hline House rent and related housing items & 36.14 & 96.2 & 96.1 & 99.0 & 104.3 & 109.8 \\
\hline Furniture and furnishings & 7.39 & 112.0 & 116.4 & 117.5 & 118.4 & 119.5 \\
\hline Medical care and health services & 1.85 & 125.7 & 132.5 & 138.8 & 150 & 151.1 \\
\hline Transportation and communication & 14.93 & 126.2 & 130.3 & 133.3 & 135.1 & 137.5 \\
\hline $\begin{array}{l}\text { Recreational, educational, } \\
\text { and cultural services }\end{array}$ & 10.29 & 114.6 & 115.2 & 125.0 & 131 & 132.4 \\
\hline Other goods and services & 8.23 & 113.0 & 116.2 & 117.0 & 117.9 & 119.5 \\
\hline
\end{tabular}

Source: Ministry of Planning.

1/ Weights are derived from 1996 Abu Dhabi household expenditure survey. 
Table 19. United Arab Emirates: Consolidated Government Finances, 1999-2003

(In millions of U.A.E. dirhams; unless otherwise stated)

\begin{tabular}{|c|c|c|c|c|c|}
\hline & 1999 & 2000 & 2001 & 2002 & $\frac{\text { Prel. }}{2003}$ \\
\hline Total revenue & 54,680 & 89,691 & 78,440 & 66,094 & 82,525 \\
\hline Hydrocarbons 1/ & 30,050 & 59,978 & 51,648 & 40,926 & 57,132 \\
\hline Non-hydrocarbon & 24,630 & 29,713 & 26,792 & 25,168 & 25,393 \\
\hline Customs & 1,886 & 1,779 & 1,846 & 1,663 & 2,323 \\
\hline Profit transfers & 3,462 & 3,936 & 3,384 & 3,357 & 3,195 \\
\hline Income tax $2 /$ & 183 & 166 & 206 & 235 & 282 \\
\hline Fees and charges & 4,581 & 4,173 & 5,120 & 6,429 & 6,845 \\
\hline Investment income 3/ & 9,714 & 15,065 & 11,576 & 8,877 & 8,246 \\
\hline Other & 4,804 & 4,586 & 4,650 & 4,607 & 4,502 \\
\hline Total expenditure and grants & 75,538 & 82,542 & 95,558 & 86,616 & 90,552 \\
\hline Current expenditure & 57,930 & 69,441 & 76,732 & 72,426 & 74,466 \\
\hline Wages and salaries 4/ 5/ & 13,224 & 13,962 & 14,383 & 15,131 & 16,105 \\
\hline Goods and services 5/ & 22,616 & 21,287 & 22,491 & 23,745 & 26,265 \\
\hline Abu Dhabi federal services 6/ & 11,899 & 19,440 & 19,082 & 17,045 & 19,157 \\
\hline Subsidies and transfers & 9,849 & 14,237 & 20,128 & 16,108 & 12,410 \\
\hline Other & 342 & 513 & 648 & 397 & 528 \\
\hline Development expenditure & 13,765 & 11,230 & 13,358 & 12,470 & 15,271 \\
\hline Loans and equity (net) & 2,435 & 652 & 4,507 & 760 & -240 \\
\hline Domestic & 2,564 & 714 & 903 & 592 & $-1,066$ \\
\hline Foreign & -129 & -62 & 3,604 & 168 & 826 \\
\hline Foreign grants $7 /$ & 1,408 & 1,219 & 961 & 960 & 1,055 \\
\hline Abu Dhabi & 1,361 & 1,071 & 664 & 784 & 925 \\
\hline Federal & 47 & 148 & 297 & 176 & 130 \\
\hline Overall balance (consolidated) & $-20,858$ & 7,149 & $-17,118$ & $-20,522$ & $-8,027$ \\
\hline (In percent of GDP) & -10.3 & 2.8 & -6.7 & -7.8 & -2.7 \\
\hline Overall balance & $-20,775$ & 32,205 & -905 & 28,377 & 40,563 \\
\hline $\begin{array}{l}\text { (Including revenues from other government entities) 8/ } \\
\text { (In percent of GDP) }\end{array}$ & -10.2 & 12.5 & -0.4 & 10.8 & 13.7 \\
\hline Financing & 20,775 & $-32,205$ & 905 & $-28,377$ & $-40,563$ \\
\hline Resident banks, net 9/ & 1,109 & $-10,530$ & -537 & $-8,483$ & $-2,269$ \\
\hline Loans to government & 3,086 & $-2,854$ & $-1,595$ & 4,292 & 5,613 \\
\hline Deposits from government & 1,977 & 7,676 & $-1,058$ & 12,775 & 7,882 \\
\hline Privatization receipts 10/ & $\ldots$ & $\ldots$ & 2,000 & $\ldots$ & 3,004 \\
\hline Change in official foreign assets ( $-=$ increase $)$ & 19,666 & $-21,675$ & -558 & $-19,894$ & $-41,298$ \\
\hline
\end{tabular}


Table 19. United Arab Emirates: Consolidated Government Finances, 1999-2003

(In millions of U.A.E. dirhams; unless otherwise stated)

\begin{tabular}{|c|c|c|c|c|c|}
\hline & 1999 & 2000 & 2001 & 2002 & $\frac{\text { Prel. }}{2003}$ \\
\hline \multicolumn{6}{|l|}{ Memorandum items: } \\
\hline Non-hydrocarbon balance 11/ & $-50,908$ & $-52,829$ & $-68,766$ & $-61,448$ & $-65,159$ \\
\hline Non-hydrocarbon revenue excluding investment income & $-60,622$ & $-67,894$ & $-80,342$ & $-70,325$ & $-73,405$ \\
\hline
\end{tabular}

Sources: Federal government; Emirate finance departments; and Fund staff estimates.

1/ Includes royalties and taxes on oil and gas companies.

2/ Taxes on profit of foreign banks.

3/ Fund staff estimates, based on fiscal accounts and other sources.

4/ Excludes military wages and salaries, which are in goods and services.

5/ Water and electricity expenditure is allocated 25 percent to wages and salaries, 75 percent goods and services.

6/ Mainly military and internal security outlays paid by Abu Dhabi, but not in federal accounts.

7/ Intergovernmental grants are netted out in the consolidated accounts.

8/ Fund staff estimates of Abu Dhabi National Oil Company (ADNOC) profits, other government entities and government domestic investments.

9/ From monetary statistics

10/ Abu Dhabi receipts from the sale of water and electricity (ADWEA) assets.

11/ Non-hydrocarbon revenues less spending. 
Table 20. United Arab Emirates: Government Current Expenditures by Economic Category and Emirate, 1999-2003

(In millions of U.A.E. dirhams)

\begin{tabular}{|c|c|c|c|c|c|}
\hline & 1999 & 2000 & 2001 & 2002 & $\frac{\text { Prel. }}{2003}$ \\
\hline Wages and salaries $1 /$ & 13,224 & 13,965 & 14,383 & 15,131 & 16,105 \\
\hline Federal & 6,922 & 7,278 & 7,468 & 7,829 & 7,841 \\
\hline Abu Dhabi 2/ & 3,531 & 3,626 & 3,528 & 3,893 & 4,435 \\
\hline Dubai 3/ & 2,328 & 2,571 & 2,885 & 2,939 & 3,331 \\
\hline Sharjah & 395 & 439 & 451 & 470 & 498 \\
\hline Ras al Khaimah 4/ & 48 & 51 & 51 & $\ldots$ & $\ldots$ \\
\hline Goods and services & 22,616 & 21,287 & 22,491 & 23,745 & 26,265 \\
\hline Federal & 8,788 & 8,668 & 8,826 & 9,216 & 9,279 \\
\hline Abu Dhabi 2/ & 10,533 & 10,459 & 11,261 & 12,148 & 14,557 \\
\hline Dubai & 2,895 & 1,740 & 1,983 & 1,971 & 2,010 \\
\hline Sharjah & 400 & 420 & 421 & 410 & 420 \\
\hline Subsidies and transfers & 9,849 & 14,237 & 20,128 & 16,108 & 12,410 \\
\hline Federal & 3,357 & 3,734 & 3,830 & 3,698 & 3,654 \\
\hline Abu Dhabi & 6,148 & 9,583 & 14,981 & 10,778 & 6,801 \\
\hline Dubai & 254 & 850 & 1,238 & 1,590 & 1,908 \\
\hline Sharjah & 52 & 30 & 39 & 42 & 47 \\
\hline Ras al Khaimah 4/ & 38 & 40 & 40 & $\ldots$ & $\ldots$ \\
\hline Other & 12,241 & 19,953 & 19,730 & 17,442 & 19,685 \\
\hline Federal & & & & & \\
\hline Abu Dhabi 5/ & 11,899 & 19,440 & 19,082 & 17,045 & 19,157 \\
\hline Dubai & 188 & 362 & 511 & 365 & 493 \\
\hline Sharjah & 40 & 35 & 21 & 32 & 35 \\
\hline Ras al Khaimah 4/ & 114 & 116 & 116 & $\ldots$ & $\ldots$ \\
\hline Total current expenditure & 57,930 & 69,441 & 76,732 & 72,426 & 74,466 \\
\hline Federal & 19,067 & 19,680 & 20,124 & 20,743 & 20,774 \\
\hline Abu Dhabi & 32,111 & 43,107 & 48,852 & 43,864 & 44,950 \\
\hline Dubai & 5,665 & 5,523 & 6,617 & 6,865 & 7,742 \\
\hline Sharjah & 887 & 924 & 932 & 954 & 1,000 \\
\hline Ras al Khaimah 4/ & 200 & 207 & 207 & $\ldots$ & $\ldots$ \\
\hline
\end{tabular}

Sources: Federal and Emirate governments.

1/ Excludes military wages and salaries.

2/ Includes pro-rated water and electricity outlays for ADWEA expenditures.

3/ Includes military wages and salaries through 1997.

4/ Data for 2000-01 estimated.

5 / Mainly federal services consisting of military and internal security outlays. 
Table 21. United Arab Emirates: Federal Government Financial Operations, 1999-2003

(In millions of U.A.E. dirhams)

\begin{tabular}{|c|c|c|c|c|c|c|}
\hline & 1999 & 2000 & 2001 & 2002 & $\frac{\text { Budget }}{2003}$ & $\frac{\text { Prel. }}{2003}$ \\
\hline Total revenue and grants & 20,268 & 20,277 & 21,008 & 21,687 & 21,071 & 21,691 \\
\hline Revenues & 6,886 & 6,965 & 7,421 & 8,508 & 7,382 & 8,636 \\
\hline Enterprise profits 1/ & 2,351 & 2,827 & 2,349 & 2,120 & 1,884 & 1,835 \\
\hline Electricity and water & 508 & 0 & 0 & 0 & 0 & 0 \\
\hline Other fees and charges & 4,027 & 4,138 & 5,072 & 6,388 & 5,498 & 6,802 \\
\hline Grants from Emirates & 13,382 & 13,312 & 13,587 & 13,179 & 13,689 & 13,055 \\
\hline Abu Dhabi & 12,182 & 12,112 & 12,387 & 11,979 & 12,489 & 11,855 \\
\hline Cash contributions & 5,600 & 5,600 & 5,600 & 5,600 & 5,600 & 5,610 \\
\hline Federal services $2 /$ & 6,571 & 6,364 & 6,343 & 6,242 & 6,629 & 6,170 \\
\hline Foreign grants on federal account 2/ & 11 & 148 & 444 & 137 & 260 & 75 \\
\hline Dubai & 1,200 & 1,200 & 1,200 & 1,200 & 1,200 & 1,200 \\
\hline Total expenditure and grants & 20,205 & 20,702 & 21,044 & 21,579 & 23,290 & 21,552 \\
\hline Current expenditures & 19,067 & 19,680 & 20,124 & 20,743 & 21,885 & 20,774 \\
\hline Wages and salaries 3/ & 6,922 & 7,278 & 7,468 & 7,829 & 8,400 & 7,841 \\
\hline Goods and services & 8,788 & 8,668 & 8,826 & 9,216 & 9,492 & 9,279 \\
\hline \multicolumn{7}{|l|}{ (By ministries) } \\
\hline (Interior and defense) 4/ & 8,953 & 8,688 & 8,796 & 9,139 & 9,381 & 9,236 \\
\hline (Education and health) & 4,771 & 5,079 & 5,243 & 5,557 & 5,813 & 5,492 \\
\hline (Other ministries) & 1,986 & 2,179 & 2,254 & 2,349 & 2,711 & 2,392 \\
\hline Subsidies and transfers 3/ & 3,357 & 3,734 & 3,830 & 3,698 & 3,993 & 3,654 \\
\hline Development expenditures & 823 & 518 & 418 & 507 & 853 & 509 \\
\hline Equity positions & 268 & 356 & 205 & 153 & 176 & 139 \\
\hline Domestic & 252 & 356 & 205 & 153 & 176 & 139 \\
\hline Foreign & 16 & 0 & 0 & 0 & 0 & 0 \\
\hline Foreign grants & 47 & 148 & 297 & 176 & 376 & 130 \\
\hline Overall balance & 63 & -425 & -36 & 108 & $-2,219$ & 140 \\
\hline \multicolumn{7}{|l|}{ Memorandum items: } \\
\hline Abu Dhabi federal services 5/ & 11,899 & 19,440 & 19,082 & 17,045 & $\ldots$ & 19,157 \\
\hline Balance on pension fund operations $6 /$ & $\ldots$ & 1,970 & 1,274 & 1,653 & $\ldots$ & $\ldots$ \\
\hline
\end{tabular}

Sources: Ministry of Finance and Industry; Abu Dhabi Finance Department; and Fund staff estimates.

1/ Dividends and payouts by Etisalat and other enterprises, including the Central Bank.

2/ Amount budgeted by federal government, but outlays are made by Abu Dhabi.

3/ Beginning 2002, military pension payments of Interior Ministry are classified as wages and salaries.

4/ Partly financed by grants from Abu Dhabi.

5/ Mainly military and internal security expenditures not included in the federal accounts.

6/ Pension fund established in 1999; not included in federal accounts. 
Table 22. United Arab Emirates: Pension Fund Operations, 2000-02 1/

(In millions of U.A.E. dirhams)

\begin{tabular}{lrrr}
\hline & 2000 & 2001 & 2002 \\
\hline Revenue & 2,131 & 1,467 & 1,926 \\
$\quad$ Employer contributions & 742 & 552 & 554 \\
Employee contributions & 246 & 184 & 185 \\
Investment income & 110 & 95 & 115 \\
Other government receipts 2/ & 500 & 0 & 0 \\
Other receipts 3/ & 533 & 636 & 1,072 \\
Expenditures & 161 & 193 & 273 \\
Pensions paid & 89 & 139 & 209 \\
Other employee benefits & 59 & 35 & 48 \\
Operating expenses & 13 & 19 & 16 \\
Other outlays & 0 & 0 & 0 \\
Balance on operations & 1,970 & 1,274 & 1,653 \\
Memorandum item: & & & \\
Assets at year-end & 1,970 & 3,244 & 4,897 \\
\end{tabular}

Source: General Pension and Social Security Authority (GPSSA).

1/ The GPSSA was established in January 1999.

2/ Initial endowment/capital from federal authorities.

3/ Transfers from Ministry of Finance and Industry, Etisalat, and others to fund pensions. 
Table 23. United Arab Emirates: Federal Subsidies and Transfers, 1999-2003

(In millions of U.A.E. dirhams)

\begin{tabular}{|c|c|c|c|c|c|c|}
\hline & 1999 & 2000 & 2001 & 2002 & $\frac{\text { Budget }}{2003}$ & $\frac{\text { Prel. }}{2003}$ \\
\hline Subsidies & 1,400 & 2,083 & 1,780 & 1,871 & 2,163 & 1,916 \\
\hline Zayed University & 0 & 420 & 190 & 210 & 209 & 209 \\
\hline U.A.E. University & 675 & 686 & 658 & 685 & 767 & 707 \\
\hline Higher College of Technology & 409 & 405 & 520 & 545 & 551 & 551 \\
\hline Electricity and water $1 /$ & 3 & 4 & 0 & 0 & 100 & 0 \\
\hline Emirates Media, Inc. & 203 & 188 & 180 & 180 & 200 & 180 \\
\hline Accumulated settlements 2/ & 0 & 0 & 19 & 0 & 15 & 15 \\
\hline Other & 110 & 380 & 213 & 251 & 321 & 254 \\
\hline Transfers & 1,957 & 1,651 & 2,050 & 1,827 & 1,399 & 1,738 \\
\hline Pension Fund 3/ & 904 & 774 & 809 & 462 & 340 & 422 \\
\hline Marriage Fund & 237 & 227 & 216 & 216 & 250 & 216 \\
\hline Zayed Housing Program 4/ & 500 & 354 & 562 & 548 & 209 & 500 \\
\hline \multicolumn{7}{|l|}{ General Pension and } \\
\hline Social Security Authority 5/ & 316 & 296 & 463 & 601 & 600 & 600 \\
\hline Total & 3,357 & 3,734 & 3,830 & 3,698 & 3,993 & 3,654 \\
\hline (In percent of GDP) & 1.7 & 1.4 & 1.5 & 1.4 & 1.4 & 1.2 \\
\hline
\end{tabular}

Source: Ministry of Finance and Industry.

1/ For federal water and power fund in northern emirates.

2/ To clear prior year budget shortfalls in universities.

3/ Beginning 2002, military pension payments are classified as wages and salaries.

4/ Law requires budget projection of $\mathrm{Dh} 640$ million.

$5 /$ Transfers to fund pension payments for federal workers retiring in current year. 
Table 24. United Arab Emirates: Federal Development Expenditures, 1999-2003

(In millions of U.A.E. dirhams)

\begin{tabular}{|c|c|c|c|c|c|c|}
\hline & 1999 & 2000 & 2001 & 2002 & $\frac{\text { Budget }}{2003}$ & $\frac{\text { Prel. }}{2003}$ \\
\hline Agriculture & 18 & 3 & 2 & 2 & 2 & 1 \\
\hline Electricity and water & 305 & 0 & 0 & 0 & 0 & 0 \\
\hline Transport and communications & 3 & 47 & 113 & 175 & 256 & 154 \\
\hline Public works and housing & 274 & 169 & 101 & 65 & 86 & 67 \\
\hline Education & 104 & 143 & 70 & 129 & 207 & 161 \\
\hline Health & 48 & 10 & 24 & 30 & 80 & 38 \\
\hline Interior and justice & 28 & 32 & 74 & 44 & 110 & 35 \\
\hline Foreign affairs & 37 & 97 & 21 & 36 & 80 & 34 \\
\hline Other & 6 & 17 & 13 & 26 & 32 & 20 \\
\hline Total & 823 & 518 & 418 & 507 & 853 & 509 \\
\hline
\end{tabular}

Source: Ministry of Finance and Industry. 
Table 25. United Arab Emirates: Abu Dhabi Fiscal Operations, 1999-2003

(In millions of U.A.E. dirhams)

\begin{tabular}{|c|c|c|c|c|c|}
\hline & 1999 & 2000 & 2001 & 2002 & $\frac{\text { Prel. }}{2003}$ \\
\hline Total revenue & 37,114 & 69,538 & 58,923 & 46,709 & 61,808 \\
\hline Hydrocarbon revenue & 25,228 & 52,591 & 45,488 & 36,045 & 51,830 \\
\hline Crude oil royalties and taxes & 23,232 & 48,633 & 42,366 & 33,692 & 48,618 \\
\hline Income taxes $1 /$ & 1,996 & 3,958 & 3,122 & 2,353 & 3,212 \\
\hline Nonhydrocarbon & 11,886 & 16,947 & 13,435 & 10,664 & 9,978 \\
\hline Customs & 319 & 262 & 276 & 294 & 491 \\
\hline Water and electricity & 12 & 0 & 0 & 1 & 0 \\
\hline Investment income $2 /$ & 9,714 & 15,065 & 11,576 & 8,877 & 8,246 \\
\hline Other & 1,841 & 1,620 & 1,583 & 1,492 & 1,241 \\
\hline Total expenditure and grants & 56,761 & 63,784 & 76,279 & 66,327 & 69,067 \\
\hline Current expenditures & 32,111 & 43,107 & 48,852 & 43,864 & 44,950 \\
\hline Wages and salaries & 2,993 & 3,135 & 3,215 & 3,374 & 3,509 \\
\hline Goods and services & 8,918 & 8,987 & 10,323 & 10,590 & 11,777 \\
\hline Federal services $3 /$ & 11,899 & 19,440 & 19,082 & 17,045 & 19,157 \\
\hline Water and electricity & 2,153 & 1,962 & 1,251 & 2,077 & 3,706 \\
\hline Subsidies and transfers & 6,148 & 9,583 & 14,981 & 10,778 & 6,801 \\
\hline Development expenditures & 8,944 & 7,452 & 10,424 & 9,203 & 11,816 \\
\hline Water and electricity & 2,121 & 1,335 & 3,259 & 1,748 & 2,766 \\
\hline Other & 6,823 & 6,117 & 7,165 & 7,455 & 9,050 \\
\hline Loans and equity (net) 4/ & 2,163 & 43 & 3,952 & 497 & -479 \\
\hline Domestic & 2,308 & 105 & 348 & 329 & $-1,305$ \\
\hline Building and housing loans & 891 & -118 & $-1,094$ & $-1,191$ & $-1,646$ \\
\hline Equity & 1,417 & 223 & 1,442 & 1,520 & 341 \\
\hline Foreign loans & -145 & -62 & 3,604 & 168 & 826 \\
\hline Grants & 13,543 & 13,182 & 13,051 & 12,763 & 12,780 \\
\hline Cash contributions to federal government & 5,600 & 5,600 & 5,600 & 5,600 & 5,610 \\
\hline Federal services $5 /$ & 6,571 & 6,364 & 6,343 & 6,242 & 6,170 \\
\hline Foreign grants on federal account $5 /$ & 11 & 147 & 444 & 137 & 75 \\
\hline Foreign grants $6 /$ & 1,361 & 1,071 & 664 & 784 & 925 \\
\hline Overall balance & $-10,127$ & 16,062 & $-17,356$ & $-19,618$ & $-7,259$ \\
\hline \multicolumn{6}{|l|}{ Memorandum items: } \\
\hline Overall balance excluding loans and equity & $-7,964$ & 16,105 & $-13,404$ & $-19,121$ & $-7,738$ \\
\hline Overall balance excluding investment income & $-19,841$ & 997 & $-28,932$ & $-28,495$ & $-15,505$ \\
\hline Privatization receipts from ADWEA 7/ & $\ldots$ & $\ldots$ & 2,000 & $\ldots$ & 3,004 \\
\hline
\end{tabular}

Source: Department of Finance of Abu Dhabi.

$1 /$ Income taxes are entirely from ADGAS and GASCO.

2/ Fund staff estimates; not included in Finance Department accounts.

3/ Mainly defense and security outlays; not included in the federal accounts.

4/ Financing items under international standards, but treated as expenditure in $\mathrm{AD}$ accounts.

5/ Outlays made by Abu Dhabi, but included in the federal accounts.

6/ Foreign grants on Abu Dhabi account.

7/ Sale of electricity and water assets of ADWEA; shown as receipts in Abu Dhabi fiscal accounts. 
Table 26. United Arab Emirates: Abu Dhabi Development Expenditures, 1999-2003

(In millions of U.A.E. dirhams)

\begin{tabular}{lrrrrr}
\hline & & & & & Prel. \\
\cline { 3 - 6 } & 1999 & 2000 & 2001 & 2002 & 2003 \\
\hline & & & & & \\
Agriculture & 688 & 907 & 845 & 681 & 736 \\
Electricity and water & 2,121 & 1,336 & 3,259 & 1,748 & 2,766 \\
Industry and commerce & 93 & 93 & 192 & 525 & 1,287 \\
Transport and communications & 2,869 & 2,272 & 2,664 & 2,570 & 1,993 \\
Housing & 1,004 & 807 & 693 & 690 & 1,933 \\
Urban development & 1,122 & 840 & 1,102 & 1,082 & 865 \\
Sewerage & 565 & 590 & 729 & 832 & 869 \\
Sports and recreation & 419 & 464 & 694 & 868 & 1,227 \\
General administration & 63 & 143 & 246 & 207 & 140 \\
& & & & & \\
Total & 8,944 & 7,452 & 10,424 & 9,203 & 11,816 \\
& & & & & \\
\hline
\end{tabular}

Source: Department of Finance of Abu Dhabi. 
Table 27. United Arab Emirates: Abu Dhabi Government Transfers and Subsidies, 1999-2003 1/

(In millions of U.A.E. dirhams)

\begin{tabular}{lrrrrr}
\hline & & & & & Prel. \\
\cline { 2 - 5 } & 1999 & 2000 & 2001 & 2002 & 2003 \\
\hline & & & & & \\
Compensation for land & 36 & 29 & 9 & 2 & 120 \\
Compensation for crop damage 1/ & 3,816 & 5,077 & 3,933 & 4,277 & 2,450 \\
Grants to sports clubs & 111 & 130 & 146 & 141 & 158 \\
Grants to low cost house owners & 8 & 36 & 13 & 24 & 45 \\
Other subsidies & 449 & 389 & 350 & 493 & 548 \\
Domestic aid 2/ & 1,692 & 3,082 & 10,803 & 5,824 & 3,480 \\
Extra-ordinary expenses 3/ & 36 & 840 & -273 & 17 & 0 \\
& & & & & \\
Total & 6,148 & 9,583 & 14,981 & 10,778 & 6,801 \\
& & & & &
\end{tabular}

Sources: Department of Finance of Abu Dhabi; and Fund staff projections.

1/ Reflecting the cost of disposition.

2/ Transfers to other emirates besides Dubai and Sharjah.

3/ The 2001 figure reflects adjustment due to overpayment in the previous year. 
Table 28. United Arab Emirates: Dubai Government Operations, 1999-2003 1/

(In millions of U.A.E. dirhams)

\begin{tabular}{|c|c|c|c|c|c|c|}
\hline & 1999 & 2000 & 2001 & 2002 & $\frac{\text { Budget }}{2003}$ & $\begin{array}{c}\text { Prel. } \\
2003\end{array}$ \\
\hline Total revenue & 8,724 & 11,079 & 10,211 & 9,103 & 9,584 & 10,091 \\
\hline Nontax revenue & 7,163 & 9,548 & 8,590 & 7,690 & 7,687 & 8,187 \\
\hline Oil and gas & 3,670 & 5,875 & 4,949 & 3,735 & 3,578 & 4,000 \\
\hline Enterprise profits $1 /$ & 1,111 & 1,111 & 1,035 & 1,237 & 1,062 & 1,361 \\
\hline Other $2 /$ & 2,382 & 2,562 & 2,606 & 2,718 & 3,047 & 2,827 \\
\hline Tax revenue & 1,561 & 1,531 & 1,621 & 1,413 & 1,897 & 1,904 \\
\hline Customs 3/ & 1,378 & 1,365 & 1,415 & 1,178 & 1,611 & 1,622 \\
\hline Income tax 4/ & 183 & 166 & 206 & 235 & 286 & 282 \\
\hline Total expenditure & 9,850 & 9,341 & 10,008 & 10,215 & 10,477 & 11,286 \\
\hline Current & 5,665 & 5,523 & 6,617 & 6,865 & 7,287 & 7,742 \\
\hline Wages and salaries & 2,328 & 2,571 & 2,885 & 2,939 & 3,298 & 3,331 \\
\hline Goods and services 2/ 5/ & 2,895 & 1,740 & 1,983 & 1,971 & 2,065 & 2,010 \\
\hline Subsidies and transfers $6 /$ & 254 & 850 & 1,238 & 1,590 & 1,410 & 1,908 \\
\hline Other & 188 & 362 & 511 & 365 & 514 & 493 \\
\hline Development & 2,981 & 2,365 & 1,841 & 2,040 & 1,980 & 2,244 \\
\hline Loans and equity (net) & 4.0 & 253.0 & 350.0 & 110.0 & 10.0 & 100.0 \\
\hline Domestic & 4.0 & 253.0 & 350.0 & 110.0 & 10.0 & 100.0 \\
\hline Foreign & 0.0 & 0.0 & 0.0 & 0.0 & 0.0 & 0.0 \\
\hline \multicolumn{7}{|l|}{ Grants } \\
\hline Contribution to federal government & 1,200 & 1,200 & 1,200 & 1,200 & 1,200 & 1,200 \\
\hline Overall balance & $-1,126$ & 1,738 & 203 & $-1,112$ & -893 & $-1,195$ \\
\hline
\end{tabular}

Source: Department of Finance of Dubai.

1/ Includes DUBAL, DUGAS, Emirates Airlines, Jebel Ali, and other public enterprises.

2 / Some years affected by timing irregularities.

3/ All revenues associated with trade and port operations; more than customs duties.

4/ Taxes on foreign banks.

5/ Includes interest and amortization on some bank loans.

6/ Excludes Water and Electricity, which is settled in an off-budget account. 
Table 29. United Arab Emirates: Sharjah Government Fiscal Operations, 1999-2003 1/ (In millions of U.A.E. dirhams)

\begin{tabular}{|c|c|c|c|c|c|c|}
\hline & 1999 & 2000 & 2001 & 2002 & $\frac{\text { Budget }}{2003}$ & $\frac{\text { Prel. }}{2003}$ \\
\hline Total revenue & 1,728 & 1,877 & 1,653 & 1,774 & 1,527 & 1,989 \\
\hline Oil and gas & 1,152 & 1,512 & 1,211 & 1,146 & 1,100 & 1,302 \\
\hline Non-oil & 576 & 365 & 442 & 628 & 427 & 687 \\
\hline Departmental receipts & 376 & 340 & 397 & 406 & 407 & 468 \\
\hline Port authority & 80 & 60 & 75 & 70 & 72 & 75 \\
\hline Customs & 189 & 152 & 155 & 191 & 155 & 210 \\
\hline Fees and charges & 28 & 28 & 41 & 40 & 40 & 43 \\
\hline Other, Of which: & 79 & 100 & 126 & 105 & 140 & 140 \\
\hline Airport & 67 & 90 & 99 & 95 & 115 & 115 \\
\hline Land sales and profit transfers & 200 & 25 & 45 & 222 & 20 & 219 \\
\hline Total expenditure & 1,832 & 1,744 & 1,532 & 1,674 & 1,577 & 1,702 \\
\hline Current & 887 & 924 & 932 & 954 & 977 & 1,000 \\
\hline Wages and salaries & 395 & 439 & 451 & 470 & 475 & 498 \\
\hline Goods and services & 400 & 420 & 421 & 410 & 432 & 420 \\
\hline Subsidies and transfers $2 /$ & 52 & 30 & 39 & 42 & 45 & 47 \\
\hline Other & 40 & 35 & 21 & 32 & 25 & 35 \\
\hline Development & 945 & 820 & 600 & 720 & 600 & 702 \\
\hline Overall balance & -104 & 133 & 121 & 100 & -50 & 287 \\
\hline
\end{tabular}

Source: Sharjah Department of Finance.

1/ Excludes operations of Sharjah Municipality.

2/ Mainly universities, does not include support for water and electricity operations. 
Table 30. United Arab Emirates: Monetary Survey, 1998-2003 1/

(In millions of U.A.E. dirhams)

\begin{tabular}{|c|c|c|c|c|c|c|}
\hline End of Period Stock & 1998 & 1999 & 2000 & 2001 & 2002 & $\frac{\text { Prel. }}{2003}$ \\
\hline Net foreign assets & 62,737 & 65,880 & 88,111 & 99,179 & 128,654 & 131,848 \\
\hline Foreign assets & 116,080 & 121,971 & 141,112 & 149,991 & 166,960 & 167,255 \\
\hline Central bank & 34,148 & 40,163 & 50,759 & 52,471 & 56,229 & 55,518 \\
\hline Commercial banks 2/ & 81,932 & 81,808 & 90,353 & 97,520 & 110,731 & 111,737 \\
\hline Foreign liabilities & 53,343 & 56,091 & 53,001 & 50,812 & 38,306 & 35,407 \\
\hline Central bank & 11 & 403 & 587 & 516 & 284 & 349 \\
\hline Commercial banks 2/ & 53,332 & 55,688 & 52,414 & 50,296 & 38,022 & 35,058 \\
\hline Domestic assets & 36,087 & 44,248 & 38,657 & 47,186 & 40,590 & 64,703 \\
\hline Claims on government (net) & $-8,807$ & $-7,698$ & $-18,228$ & $-18,765$ & $-27,248$ & $-29,517$ \\
\hline Claims & 11,568 & 14,654 & 11,800 & 10,205 & 14,497 & 20,110 \\
\hline Deposits & 20,375 & 22,352 & 30,028 & 28,970 & 41,745 & 49,627 \\
\hline Claims on public sector enterprises & 5,236 & 5,581 & 5,780 & 5,258 & 7,122 & 12,990 \\
\hline Claims on private nonbanks & 105,895 & 113,430 & 123,313 & 134,132 & 149,352 & 169,469 \\
\hline Capital and reserves & $-31,430$ & $-33,517$ & $-35,833$ & $-38,377$ & $-42,583$ & $-46,063$ \\
\hline Other items (net) & $-34,807$ & $-33,548$ & $-36,375$ & $-35,062$ & $-46,053$ & $-42,176$ \\
\hline Central bank & $-13,933$ & $-16,261$ & $-26,292$ & $-27,994$ & $-31,245$ & $-28,416$ \\
\hline Commercial banks & $-20,874$ & $-17,287$ & $-10,083$ & $-7,068$ & $-14,808$ & $-13,760$ \\
\hline Domestic liquidity & 98,824 & 110,128 & 126,768 & 146,369 & 169,244 & 196,551 \\
\hline Money & 27,783 & 30,250 & 34,067 & 39,464 & 47,054 & 58,262 \\
\hline Currency outside banks & 8,194 & 10,270 & 10,017 & 10,537 & 11,938 & 13,785 \\
\hline Dirham demand deposits & 19,589 & 19,980 & 24,050 & 28,927 & 35,116 & 44,477 \\
\hline Quasi-money & 71,041 & 79,878 & 92,701 & 106,905 & 122,190 & 138,289 \\
\hline Foreign currency deposits & 19,731 & 23,354 & 28,196 & 33,078 & 39,605 & 46,295 \\
\hline Dirham time and savings deposits & 51,310 & 56,524 & 64,505 & 73,827 & 82,585 & 91,994 \\
\hline \multicolumn{7}{|l|}{ Memorandum items: } \\
\hline Dirham-denominated liquidity & 70,899 & 76,504 & 88,555 & 102,754 & 117,701 & 137,632 \\
\hline Change in percent & 2.9 & 7.9 & 15.8 & 7.3 & 14.5 & 16.9 \\
\hline Foreign currency deposits of residents & 27,925 & 33,624 & 38,213 & 43,615 & 51,543 & 58,919 \\
\hline Change in percent & 7.7 & 20.4 & 13.6 & -4.7 & 18.2 & 14.3 \\
\hline $\begin{array}{l}\text { Ratio of foreign currency deposits to } \\
\text { total deposits (in percent) }\end{array}$ & 30.8 & 33.7 & 32.7 & 32.1 & 32.8 & 32.0 \\
\hline
\end{tabular}

Source: Central Bank of the United Arab Emirates.

1/ Compiled in accordance with the residence principle.

$2 /$ Including the restricted license bank. 
Table 31. United Arab Emirates: Factors Affecting Domestic Liquidity, 1998-2003

(Annual changes in millions of U.A.E. dirhams)

\begin{tabular}{|c|c|c|c|c|c|c|}
\hline End of Period & 1998 & 1999 & 2000 & 2001 & 2002 & $\frac{\text { Prel. }}{2003}$ \\
\hline Net foreign assets & 372 & 3,143 & 22,231 & 11,068 & 29,475 & 3,194 \\
\hline Foreign assets & 7,929 & 5,891 & 19,141 & 8,879 & 16,969 & 295 \\
\hline Central bank & 2,717 & 6,015 & 10,596 & 1,712 & 3,758 & -711 \\
\hline Commercial banks & 5,212 & -124 & 8,545 & 7,167 & 13,211 & 1,006 \\
\hline Foreign liabilities & 7,557 & 2,748 & $-3,090$ & $-2,189$ & $-12,506$ & $-2,899$ \\
\hline Central bank & -41 & 392 & 184 & -71 & -232 & 65 \\
\hline Commercial banks $1 /$ & 7,598 & 2,356 & $-3,274$ & $-2,118$ & $-12,274$ & $-2,964$ \\
\hline Domestic assets & 3,599 & 8,161 & $-5,591$ & 8,529 & $-6,596$ & 24,113 \\
\hline Claims on government (net) & $-1,248$ & 1,109 & $-10,530$ & -537 & $-8,483$ & $-2,269$ \\
\hline Claims & 3,469 & 3,086 & $-2,854$ & $-1,595$ & 4,292 & 5,613 \\
\hline Deposits & 4,717 & 1,977 & 7,676 & $-1,058$ & 12,775 & 7,882 \\
\hline Claims on public sector enterprises & -275 & 345 & 199 & -522 & 1,864 & 5,868 \\
\hline Claims on private nonbanks & 13,160 & 7,535 & 9,883 & 10,819 & 15,220 & 20,117 \\
\hline Capital and reserves & $-4,449$ & $-2,087$ & $-2,316$ & $-2,544$ & $-4,206$ & $-3,480$ \\
\hline Other items (net) & $-3,589$ & 1,259 & $-2,827$ & 1,313 & $-10,991$ & 3,877 \\
\hline Central bank & $-1,027$ & $-2,328$ & $-10,031$ & $-1,702$ & $-3,251$ & 2,829 \\
\hline Commercial banks & $-2,562$ & 3,587 & 7,204 & 3,015 & $-7,740$ & 1,048 \\
\hline Domestic liquidity & 3,970 & 11,304 & 16,640 & 19,601 & 22,875 & 27,307 \\
\hline Money & 2,415 & 2,467 & 3,817 & 5,397 & 7,590 & 11,208 \\
\hline Currency outside banks & 828 & 2,076 & -253 & 520 & 1,401 & 1,847 \\
\hline Dirham demand deposits & 1,587 & 391 & 4,070 & 4,877 & 6,189 & 9,361 \\
\hline Quasi-money & 1,555 & 8,837 & 12,823 & 14,204 & 15,285 & 16,099 \\
\hline Foreign currency deposits & 1,161 & 3,623 & 4,842 & 4,882 & 6,527 & 6,690 \\
\hline Dirham time and savings deposits & 394 & 5,214 & 7,981 & 9,322 & 8,758 & 9,409 \\
\hline
\end{tabular}

Source: Central Bank of the United Arab Emirates.

1/ Including the restricted license bank. 
Table 32. United Arab Emirates: Summary Accounts of the Central Bank, 1998-2003 (In millions of U.A.E. dirhams)

\begin{tabular}{|c|c|c|c|c|c|c|}
\hline End of Period & 1998 & 1999 & 2000 & 2001 & 2002 & 2003 \\
\hline Foreign assets & 34,148 & 40,163 & 50,759 & 52,471 & 56,229 & 55,518 \\
\hline Claims on banks & 23,086 & 21,218 & 28,662 & 31,108 & 49,195 & 42,746 \\
\hline Loans and investments & 8,724 & 16,885 & 20,190 & 20,000 & 5,509 & 11,348 \\
\hline Other $1 /$ & 1,187 & 989 & 1,126 & 533 & 372 & 127 \\
\hline IMF reserve position & 1,151 & 1,071 & 781 & 830 & 1,153 & 1,297 \\
\hline Net claims on government & $-1,151$ & $-1,071$ & -781 & -830 & $-1,153$ & $-1,297$ \\
\hline Claims & 0 & 0 & 0 & 0 & & \\
\hline Less: IMF reserve position & 1,151 & 1,071 & 781 & 830 & 1,153 & 1,297 \\
\hline Claims on public sector enterprises & 0 & 0 & & 0 & & \\
\hline Claims on private nonbanks $2 /$ & 19 & 21 & 24 & 29 & 62 & 75 \\
\hline Claims on commercial banks & 50 & 50 & 50 & 50 & 50 & 50 \\
\hline Unclassified assets & 72 & 70 & 84 & 118 & 85 & 156 \\
\hline Total assets/liabilities & 33,138 & 39,233 & 50,136 & 51,838 & 55,273 & 54,502 \\
\hline Foreign liabilities & 11 & 403 & 587 & 516 & 284 & 349 \\
\hline Reserve money & 16,233 & 20,099 & 20,288 & 21,608 & 25,160 & 30,539 \\
\hline Currency outside banks & 8,194 & 10,270 & 10,017 & 10,537 & 11,938 & 13,785 \\
\hline Cash held by banks & 1,426 & 2,624 & 2,228 & 1,783 & 1,861 & 2,184 \\
\hline Banks' deposits & 6,613 & 7,205 & 8,043 & 9,288 & 11,361 & 14,570 \\
\hline Certificates of deposit & 4,092 & 6,429 & 15,624 & 16,779 & 12,489 & 11,762 \\
\hline Government deposits 3/ & 9,378 & 10,619 & 11,546 & 11,063 & 10,111 & 10,186 \\
\hline Capital and reserves & 1,500 & 1,560 & 1,560 & 1560 & 1560 & 1,560 \\
\hline Unclassified liabilities 4/ & 1924 & 123 & 531 & 312 & 5669 & 106 \\
\hline
\end{tabular}

Source: Central Bank of the United Arab Emirates.

1/ Mainly gold, valued at cost.

2/ Staff loans.

3/ Mainly foreign currency deposits.

4/ Includes undistributed profits. 
Table 33. United Arab Emirates: Balance Sheets of Commercial Banks, 1998-2003 1/

(In millions of U.A.E. dirhams)

\begin{tabular}{lrrrrrr}
\hline & & & & & & Prel. \\
\cline { 3 - 6 } End of Period & 1998 & 1999 & 2000 & 2001 & 2002 & 2003 \\
& & & & & & \\
\hline & & & & & & \\
Reserves & 8,035 & 9,827 & 10,271 & 11,071 & 13,222 & 16,753 \\
$\quad$ Cash & 1,426 & 2,624 & 2,228 & 1,783 & 1,861 & 2,184 \\
$\quad$ Deposits with central bank & 6,609 & 7,203 & 8,043 & 9,288 & 11,361 & 14,569 \\
Foreign assets & 81,195 & 80,833 & 89,810 & 96,618 & 110,675 & 111,727 \\
Claims on government & 12,719 & 15,725 & 12,581 & 11,035 & 15,650 & 21,407 \\
Claims on public sector enterprises 2/ & 5,236 & 5,581 & 5,780 & 5,258 & 7,122 & 12,990 \\
Claims on private nonbanks & 102,416 & 110,276 & 119,828 & 130,549 & 145,592 & 165,143 \\
Claims on nonbank financial institutions & 3,443 & 3,075 & 3,317 & 3,415 & 3,692 & 4,251 \\
Central bank certificates of deposit & 4,092 & 6,429 & 15,624 & 16,779 & 12,489 & 11,762 \\
Unclassified assets 3/ & 6,499 & 6,632 & 7,111 & 7,230 & 6,938 & 7,390 \\
$\quad$ Total assets/liabilities & 223,635 & 238,378 & 264,322 & 281,955 & 315,380 & 351,423 \\
& & & & & & \\
Monetary deposits & 19,589 & 19,980 & 24,050 & 28,927 & 35,116 & 44,477 \\
Quasi monetary deposits & 71,001 & 79,847 & 92,670 & 106,870 & 122,183 & 138,289 \\
Foreign liabilities 4/ & 53,055 & 54,657 & 51,905 & 49,501 & 37,972 & 35,058 \\
Government deposits & 10,920 & 11,671 & 18,441 & 17,870 & 31,606 & 39,418 \\
Government lending funds & 77 & 62 & 41 & 37 & 28 & 23 \\
Credit from central bank & 51 & 54 & 52 & 55 & 61 & 101 \\
Capital and reserves & 29,883 & 31,910 & 34,226 & 36,769 & 40,975 & 44,455 \\
Provision & $\ldots$ & 29,216 & 29,360 & 30,054 & 32,246 & 31983 \\
Unclassified liabilities & 39,059 & 10,981 & 13,577 & 11,872 & 15,193 & 17,619 \\
& & & & & & \\
\hline & & & & & &
\end{tabular}

Source: Central Bank of the United Arab Emirates.

1/ Excluding accounts of the restricted license bank.

2/ Commercial enterprises with significant government ownership, including Dubai Aluminum Company, Dubai Gas Company, Abu Dhabi National Oil Company, other oil and gas companies owned by

Abu Dhabi, and cement companies established by several Emirate governments.

3 / Includes net lending to restricted license bank.

4/ Includes commercial prepayments. 
Table 34. United Arab Emirates: Balance Sheet of Restricted License Bank, 1998-2003 1/

(In millions of U.A.E. dirhams)

\begin{tabular}{|c|c|c|c|c|c|c|}
\hline End of Period & 1998 & 1999 & 2000 & 2001 & 2002 & $\frac{\text { Prel. }}{2003}$ \\
\hline Reserves & 4 & 3 & 3 & 5 & 6 & 0 \\
\hline Cash & 0 & 0 & 0 & 0 & 0 & 0 \\
\hline Deposits with central bank & 4 & 3 & 3 & 5 & 6 & 0 \\
\hline Foreign assets & 737 & 975 & 543 & 902 & 56 & 10 \\
\hline Claims on government & 0 & 0 & 0 & 0 & 0 & 0 \\
\hline Claims on public sector enterprises & 0 & 0 & 0 & 0 & 0 & 0 \\
\hline Claims on private nonbanks & 17 & 58 & 144 & 139 & 6 & 0 \\
\hline Claims on banks & 136 & 249 & 113 & 110 & 22 & 20 \\
\hline Other assets & 2 & 1 & 1 & 1 & 18 & 19 \\
\hline Total assets/liabilities & 896 & 1,286 & 804 & 1157 & 108 & 49 \\
\hline Foreign liabilities & 277 & 1,031 & 509 & 795 & 50 & 0 \\
\hline Quasi monetary deposits 2/ & 40 & 31 & 31 & 35 & 7 & 0 \\
\hline Government deposits & 0 & 0 & 0 & 0 & 0 & 0 \\
\hline Liabilities to banks & 523 & 167 & 207 & 269 & 0 & 0 \\
\hline Capital and reserves & 47.0 & 47.0 & 47.0 & 48 & 48 & 48 \\
\hline Other liabilities & 9 & 10 & 10 & 10 & 3 & 1 \\
\hline
\end{tabular}

Source: Central Bank of the United Arab Emirates.

1/ Banca Commercial Italiana was the only restricted bank, it stopped all operations on May 31, 2003.

2/ Foreign currency deposits. 
Table 35. United Arab Emirates: Licensed Commercial Banks, December 2003

(In millions of U.A.E. dirhams)

\begin{tabular}{|c|c|c|c|}
\hline & $\begin{array}{c}\text { Head Office and } \\
\text { Branches }\end{array}$ & $\begin{array}{c}\text { Year } \\
\text { Established }\end{array}$ & $\begin{array}{c}\text { Balance } \\
\text { Sheet } \\
\text { (Dh millions) }\end{array}$ \\
\hline Abu Dhabi Commercial Bank & 38 & 1985 & 25,717 \\
\hline Abu Dhabi Islamic Bank & 12 & 1997 & 8,548 \\
\hline Arbift & 5 & 1976 & 3,596 \\
\hline Bank of Sharjah & 3 & 1974 & 2,689 \\
\hline Commercial Bank International & 7 & 1991 & 2,978 \\
\hline Commercial Bank of Dubai & 21 & 1969 & 7,298 \\
\hline Dubai Bank & 2 & 2002 & 835 \\
\hline Dubai Islamic Bank & 17 & 1975 & 21,866 \\
\hline Emirates Bank International & 26 & 1977 & 27,366 \\
\hline First Gulf Bank & 5 & 1979 & 5,985 \\
\hline InvestBank & 5 & 1975 & 3,000 \\
\hline Mashreq Bank & 32 & 1967 & 20,595 \\
\hline Middle East Bank (sub of EBI) & 13 & 1976 & 2,100 \\
\hline National Bank of Abu Dhabi & 57 & 1968 & 30,374 \\
\hline National Bank of Dubai & 33 & 1963 & 31,070 \\
\hline National Bank of Fujairah & 6 & 1384 & 2,919 \\
\hline National Bank of Ras Al Khaimah & 13 & 1976 & 3,139 \\
\hline National Bank of Sharjah & 10 & 1976 & 2,640 \\
\hline National Bank of Umm Al Qaiwain & 10 & 1982 & 1,778 \\
\hline Union National Bank & 29 & 1982 & 15,270 \\
\hline United Arab Bank & 9 & 1975 & 2,458 \\
\hline Total U.A.E. banks & 353 & & 222,221 \\
\hline
\end{tabular}

Source: Central Bank of the United Arab Emirates. 
Table 36. United Arab Emirates: Balance of Payments, 1998-2003

(In billions of U.S. dollars)

\begin{tabular}{|c|c|c|c|c|c|c|}
\hline & 1998 & 1999 & 2000 & 2001 & 2002 & $\frac{\text { Prel. }}{2003}$ \\
\hline Trade balance & 4.7 & 8.6 & 18.8 & 14.0 & 14.5 & 19.1 \\
\hline Exports & 33.4 & 36.5 & 49.6 & 47.5 & 51.2 & 60.8 \\
\hline Oil and products & 10.4 & 14.2 & 23.4 & 19.7 & 19.7 & 25.7 \\
\hline Crude oil and condensates & 10.0 & 13.6 & 21.7 & 17.6 & 16.7 & 22.1 \\
\hline Petroleum products $1 /$ & 0.4 & 0.6 & 1.7 & 2.1 & 3.0 & 3.5 \\
\hline Gas & 2.1 & 2.3 & 3.7 & 3.3 & 3.1 & 3.9 \\
\hline Non-hydrocarbon & 6.2 & 6.3 & 7.5 & 8.5 & 10.6 & 11.3 \\
\hline Exports by Emirates & 1.9 & 1.8 & 2.0 & 2.0 & 2.4 & 2.5 \\
\hline Free zone exports & 4.3 & 4.5 & 5.5 & 6.4 & 8.3 & 8.8 \\
\hline Re-exports, $\mathrm{O} f$ which : 2/ & 14.7 & 13.7 & 15.0 & 16.0 & 17.8 & 20.0 \\
\hline Non-monetary gold & 3.1 & 1.8 & 1.9 & 1.9 & 1.9 & 2.0 \\
\hline Imports (f.o.b.) & -28.7 & -27.9 & -30.8 & -33.5 & -36.7 & -41.7 \\
\hline Imports by Emirates, $\mathrm{O} f$ which : & -25.5 & -24.7 & -25.9 & -26.8 & -28.9 & -33.1 \\
\hline Non-monetary gold & -3.2 & -1.9 & -2.0 & -2.2 & -2.2 & -2.2 \\
\hline Unrecorded govt. imports & -0.7 & -1.6 & -1.6 & -1.9 & -1.6 & -1.6 \\
\hline Free zones & -3.2 & -3.2 & -4.9 & -6.7 & -7.9 & -8.7 \\
\hline Income, net & 5.8 & 2.2 & 3.8 & 2.9 & 0.8 & -0.1 \\
\hline Banking system & 1.0 & 1.1 & 1.6 & 1.0 & 0.5 & 0.4 \\
\hline Private non-banks & 0.4 & 0.4 & 0.4 & 0.3 & 0.1 & 0.1 \\
\hline Official & 5.0 & 2.5 & 4.0 & 3.0 & 2.2 & 1.9 \\
\hline Foreign partners - oil 3/ & -0.5 & -1.8 & -1.9 & -1.1 & -1.9 & -2.3 \\
\hline Foreign partners - gas $3 /$ & -0.1 & -0.1 & -0.3 & -0.2 & -0.2 & -0.2 \\
\hline Services, net & -5.9 & -5.9 & -6.4 & -6.2 & -7.3 & -7.5 \\
\hline Credits & 1.9 & 2.1 & 2.2 & 2.4 & 2.4 & 2.8 \\
\hline Travel & 0.9 & 1.0 & 1.1 & 1.2 & 1.2 & 1.4 \\
\hline Transport & 0.7 & 0.8 & 0.8 & 0.8 & 0.9 & 0.9 \\
\hline Government services & 0.3 & 0.3 & 0.3 & 0.4 & 0.3 & 0.4 \\
\hline Debits & -7.9 & -8.0 & -8.6 & -8.5 & -9.8 & -10.3 \\
\hline Travel & -2.8 & -2.9 & -3.0 & -3.0 & -3.7 & -3.7 \\
\hline Transport & -1.0 & -1.0 & -1.1 & -1.1 & -1.1 & -1.2 \\
\hline Government services & -0.2 & -0.2 & -0.3 & -0.3 & -0.4 & -0.4 \\
\hline Freight 4/ & -3.9 & -3.8 & -4.2 & -4.0 & -4.6 & -5.0 \\
\hline Transfers, net & -3.7 & -3.9 & -4.0 & -4.2 & -4.4 & -4.7 \\
\hline Private & -3.4 & -3.6 & -3.7 & -3.9 & -4.1 & -4.4 \\
\hline Official & -0.2 & -0.3 & -0.3 & -0.3 & -0.3 & -0.3 \\
\hline
\end{tabular}


Table 36. United Arab Emirates: Balance of Payments, 1998-2003

(In billions of U.S. dollars)

\begin{tabular}{|c|c|c|c|c|c|c|}
\hline & 1998 & 1999 & 2000 & 2001 & 2002 & $\frac{\text { Prel. }}{2003}$ \\
\hline Current account & 0.9 & 0.9 & 12.2 & 6.5 & 3.5 & 6.8 \\
\hline (In percent of GDP) & 1.8 & 1.6 & 17.3 & 9.4 & 4.9 & 8.5 \\
\hline Capital account & 0.0 & 0.0 & 0.0 & 0.0 & 0.0 & 0.0 \\
\hline Financial account & 2.9 & 7.5 & -9.7 & -1.5 & -10.7 & -10.3 \\
\hline Private capital & -0.2 & 2.1 & -3.8 & -1.3 & -5.3 & 0.9 \\
\hline Direct investment, net 5/ & 0.2 & 0.9 & -1.6 & -0.2 & -0.3 & 0.1 \\
\hline Abroad & 0.0 & -0.1 & -2.1 & -0.4 & -0.4 & -0.1 \\
\hline In reporting economy & 0.3 & 1.0 & 0.5 & 0.3 & 0.1 & 0.2 \\
\hline Portfolio securities & $\ldots$ & $\ldots$ & $\ldots$ & $\ldots$ & 0.2 & 0.0 \\
\hline Commercial banks & 0.6 & 0.7 & -3.2 & -2.5 & -6.9 & -1.1 \\
\hline Private non-banks (BIS source) & -1.1 & 0.6 & 1.0 & 1.4 & 1.7 & 1.9 \\
\hline Official capital 6/ & 3.1 & 5.4 & -5.9 & -0.2 & -5.4 & -11.2 \\
\hline Errors and omissions & -3.0 & -6.9 & 0.3 & -4.6 & 8.3 & 3.3 \\
\hline (In percent of GDP) & -6.3 & -12.4 & 0.5 & -6.6 & 11.6 & 4.1 \\
\hline Overall balance & 0.8 & 1.5 & 2.8 & 0.5 & 1.1 & -0.2 \\
\hline Central Bank net foreign assets & -0.8 & -1.5 & -2.8 & -0.5 & -1.1 & 0.2 \\
\hline \multicolumn{7}{|l|}{ Memorandum items: } \\
\hline Overall balance (in percent of GDP) & 1.6 & 2.8 & 4.0 & 0.7 & 1.5 & -0.3 \\
\hline Gross reserves of the Central Bank & 9.3 & 10.9 & 13.8 & 14.3 & 15.3 & 15.1 \\
\hline (In months of imports) 7/ & 4.0 & 4.3 & 4.9 & 4.7 & 4.4 & 4.1 \\
\hline
\end{tabular}

Sources: U.A.E. authorities; and Fund staff estimates.

1/ Includes fertilizers and lubricants.

2/ Not formally compiled; estimated at 40-50 percent of emirates imports.

3/ Fund staff estimates based on foreign partner share of oil and gas sector net profits.

4/ Estimated freight to adjust imports (cif basis in UAE BOP accounts) to fob basis.

5/ UNCTAD direct investment estimates (from World Investment Report, 2003 ).

6/ Includes changes in government external assets.

7/ Imports of goods and services in the next 12 months. 
Table 37. United Arab Emirates: Balance of Payments, 1998-2003

(In billions of U.A.E. dirhams)

\begin{tabular}{|c|c|c|c|c|c|c|}
\hline & 1998 & 1999 & 2000 & 2001 & 2002 & $\frac{\text { Prel. }}{2003}$ \\
\hline Trade balance & 17.3 & 31.6 & 68.9 & 51.5 & 53.3 & 70.2 \\
\hline Exports & 122.6 & 134.1 & 182.0 & 174.6 & 188.2 & 223.5 \\
\hline Oil and products & 38.2 & 52.1 & 85.9 & 72.5 & 72.4 & 94.2 \\
\hline Crude oil and condensates & 36.7 & 49.9 & 79.7 & 64.7 & 61.3 & 81.2 \\
\hline Petroleum products $1 /$ & 1.5 & 2.2 & 6.2 & 7.8 & 11.2 & 13.0 \\
\hline Gas & 7.8 & 8.4 & 13.5 & 12.1 & 11.4 & 14.4 \\
\hline Non-hydrocarbon exports & 22.7 & 23.3 & 27.4 & 31.1 & 39.1 & 41.6 \\
\hline Exports by Emirates & 6.9 & 6.7 & 7.2 & 7.5 & 8.7 & 9.2 \\
\hline Free zone exports & 15.8 & 16.6 & 20.2 & 23.6 & 30.4 & 32.4 \\
\hline Re-exports, Of which: 2/ & 53.9 & 50.2 & 55.2 & 58.9 & 65.2 & 73.3 \\
\hline Non-monetary gold & 11.3 & 6.7 & 7.0 & 7.0 & 7.1 & 7.3 \\
\hline Imports (f.o.b.) & -105.3 & -102.5 & -113.1 & -123.1 & -134.9 & -153.3 \\
\hline Imports by Emirates, Of which: & -93.7 & -90.6 & -95.1 & -98.5 & -106.0 & -121.5 \\
\hline Non-monetary gold & -11.7 & -6.8 & -7.2 & -7.9 & -7.9 & -7.9 \\
\hline Unrecorded govt.imports & -2.5 & -5.9 & -5.9 & -7.0 & -5.9 & -5.9 \\
\hline Free zone imports & -11.6 & -11.9 & -18.0 & -24.6 & -28.9 & -31.8 \\
\hline Income, net & 21.4 & 7.9 & 13.9 & 10.7 & 2.9 & -0.4 \\
\hline Banking system & 3.8 & 4.0 & 5.9 & 3.7 & 1.9 & 1.5 \\
\hline Private non-banks & 1.6 & 1.5 & 1.4 & 1.1 & 0.5 & 0.3 \\
\hline Official & 18.2 & 9.4 & 14.8 & 10.8 & 8.1 & 7.1 \\
\hline Foreign partners - oil 3/ & -1.9 & -6.4 & -7.2 & -4.2 & -7.1 & -8.5 \\
\hline Foreign partners - gas $3 /$ & -0.4 & -0.5 & -1.0 & -0.8 & -0.6 & -0.8 \\
\hline Services, net & -21.9 & -21.8 & -23.6 & -22.6 & -27.0 & -27.6 \\
\hline Credits & 7.1 & 7.6 & 8.0 & 8.7 & 8.9 & 10.2 \\
\hline Travel & 3.4 & 3.7 & 3.9 & 4.4 & 4.5 & 5.3 \\
\hline Transport & 2.7 & 2.8 & 2.9 & 3.0 & 3.2 & 3.4 \\
\hline Government services & 1.1 & 1.1 & 1.2 & 1.3 & 1.2 & 1.6 \\
\hline Debits & -29.0 & -29.4 & -31.5 & -31.3 & -35.9 & -37.8 \\
\hline Travel & -10.2 & -10.8 & -11.1 & -11.2 & -13.5 & -13.5 \\
\hline Transport & -3.6 & -3.7 & -3.9 & -4.0 & -4.0 & -4.4 \\
\hline Government services & -0.9 & -0.9 & -1.1 & -1.3 & -1.5 & -1.5 \\
\hline Freight $4 /$ & -14.4 & -14.0 & -15.5 & -14.9 & -16.9 & -18.4 \\
\hline Transfers (net) & -13.5 & -14.4 & -14.6 & -15.5 & -16.2 & -17.1 \\
\hline Private & -12.6 & -13.4 & -13.5 & -14.4 & -15.2 & -16.1 \\
\hline Public & -0.9 & -1.0 & -1.1 & -1.1 & -1.0 & -1.0 \\
\hline
\end{tabular}


Table 37. United Arab Emirates: Balance of Payments, 1998-2003

(In billions of U.A.E. dirhams)

\begin{tabular}{|c|c|c|c|c|c|c|}
\hline & 1998 & 1999 & 2000 & 2001 & 2002 & $\frac{\text { Prel. }}{2003}$ \\
\hline Current account & 3.3 & 3.3 & 44.6 & 24.0 & 13.0 & 25.1 \\
\hline In percent of GDP & 1.8 & 1.6 & 17.3 & 9.4 & 4.9 & 8.5 \\
\hline Capital account & 0.0 & 0.0 & 0.0 & 0.0 & 0.0 & 0.0 \\
\hline Financial account & 10.7 & 27.5 & -35.5 & -5.3 & -39.4 & -37.9 \\
\hline Private capital & -0.9 & 7.8 & -13.8 & -4.8 & -19.5 & 3.3 \\
\hline Direct investment, net 5/ & 0.8 & 3.2 & -5.8 & -0.7 & -1.3 & 0.4 \\
\hline Abroad & -0.1 & -0.4 & -7.7 & -1.6 & -1.6 & -0.4 \\
\hline In reporting economy & 0.9 & 3.6 & 1.9 & 0.9 & 0.3 & 0.7 \\
\hline Portfolio securities & $\ldots$ & $\ldots$ & $\ldots$ & $\ldots$ & 0.9 & 0.0 \\
\hline Commercial banks & 2.4 & 2.5 & -11.8 & -9.3 & -25.5 & -4.0 \\
\hline Private nonbanks (BIS source) & -4.1 & 2.1 & 3.8 & 5.2 & 6.4 & 6.9 \\
\hline Official capital 6/ & 11.6 & 19.7 & -21.7 & -0.6 & -19.9 & -41.3 \\
\hline Errors and omissions & -11.2 & -25.2 & 1.3 & -16.9 & 30.4 & 12.1 \\
\hline (In percent of GDP) & -6.3 & -12.4 & 0.5 & -6.6 & 11.6 & 4.1 \\
\hline Overall balance & 2.8 & 5.6 & 10.4 & 1.8 & 4.0 & -0.8 \\
\hline Central Bank net foreign assets & -2.8 & -5.6 & -10.4 & -1.8 & -4.0 & 0.8 \\
\hline \multicolumn{7}{|l|}{ Memorandum items: } \\
\hline Overall balance (in percent of GDP) & 1.6 & 2.8 & 4.0 & 0.7 & 1.5 & -0.3 \\
\hline Gross reserves of the Central Bank & 34.1 & 40.2 & 50.8 & 52.5 & 56.2 & 55.5 \\
\hline (In months of imports) 7/ & 4.0 & 4.3 & 4.9 & 4.7 & 4.4 & 4.1 \\
\hline
\end{tabular}

Source: U.A.E. authorities; and Fund staff estimates.

$1 /$ Includes fertilizers and lubricants.

2 / Not formally compiled; estimated at 40-50 percent of emirates imports.

3/ Fund staff estimates based on foreign partner share of oil and gas sector net profits.

4/ Estimated freight to adjust imports (cif basis in UAE BOP accounts) to fob basis.

5/ UNCTAD direct investment estimates (from World Investment Report, 2003 ).

$6 /$ Includes changes in government external assets.

7/ Imports of goods and services in the next 12 months. 
Table 38. United Arab Emirates: Merchandise Imports by Harmonized System Sections, 1998-2002 1/

(In millions of U.A.E. dirhams)

\begin{tabular}{|c|c|c|c|c|c|}
\hline & 1998 & 1999 & 2000 & 2001 & 2002 \\
\hline Live animals, animal products & 2,774 & 2,834 & 2,900 & 3,314 & 3,006 \\
\hline Vegetable products & 4,854 & 4,815 & 5,135 & 5,831 & 5,515 \\
\hline Fats, oil and waxes & 415 & 413 & 451 & 461 & 345 \\
\hline Foodstuffs, beverages, spirits, and tobacco & 2,554 & 2,578 & 2,540 & 3,387 & 3,336 \\
\hline Mineral products & 1,549 & 1,605 & 1,922 & 2,209 & 1,171 \\
\hline Chemicals and related materials & 5,897 & 5,750 & 5,998 & 6,914 & 6,970 \\
\hline Plastics and rubber & 3,790 & 3,668 & 3,760 & 4,810 & 4,663 \\
\hline Raw hides, leather, and articles thereof & 566 & 550 & 509 & 737 & 559 \\
\hline Wood, cork, and articles thereof & 1,027 & 1,009 & 959 & 1,525 & 1,121 \\
\hline Wood pulp, paper, and paperboard & 1,386 & 1,302 & 1,330 & 1,714 & 1,562 \\
\hline Textiles and textile articles & 11,126 & 10,914 & 10,642 & 15,322 & 10,378 \\
\hline Footwear and other accessories & 1,183 & 1,238 & 1,017 & 1,813 & 1,057 \\
\hline Stone, plaster, cement, ceramic, and glassware & 2,035 & 2,036 & 2,103 & 2,620 & 2,487 \\
\hline Pearls, precious stones, and precious metals $2 /$ & 2,673 & 2,925 & 3,499 & 2,481 & 17,357 \\
\hline Base metals and related products & 8,306 & 8,263 & 8,587 & 9,267 & 9,718 \\
\hline Machinery and electrical equipment & 22,691 & 22,845 & 24,871 & 26,067 & 28,957 \\
\hline Vehicles and other transport equipment & 11,720 & 13,069 & 15,880 & 15,464 & 14,764 \\
\hline Optical and medical equipment & 2,765 & 2,852 & 2,867 & 3,070 & 3,270 \\
\hline Arms and ammunition & 224 & 9 & 23 & 24 & 25 \\
\hline Miscellaneous manufactured goods & 3,178 & 2,944 & 3,007 & 3,302 & 3,104 \\
\hline Works of art and antiques & 109 & 92 & 119 & 122 & 124 \\
\hline Total imports, c.i.f. & 90,822 & 91,711 & 98,119 & 110,454 & 119,489 \\
\hline
\end{tabular}

Source: Central Bank of the United Arab Emirates.

1/ Imports of the Emirates of Abu Dhabi, Dubai, and Sharjah.

2/ As from 2002 imports of nonmonetary gold included by Dubai authorities. Exports of nonmonetary gold are classified as "re-exports." 
Table 39. United Arab Emirates: Merchandise Exports by

Harmonized System Sections, 1999-2002 1/

(In millions of U.A.E. dirhams)

\begin{tabular}{|c|c|c|c|c|}
\hline & 1999 & 2000 & 2001 & 2002 \\
\hline Live animals, animal products & 122 & 102 & 201 & 126 \\
\hline Vegetable products & 134 & 112 & 185 & 156 \\
\hline Fats, oil and waxes & 136 & 137 & 168 & 158 \\
\hline Foodstuffs, beverages, spirits, and tobacco & 309 & 343 & 365 & 459 \\
\hline Mineral products & 297 & 349 & 335 & 396 \\
\hline Chemicals and related materials & 297 & 293 & 422 & 398 \\
\hline Plastics and rubber & 131 & 160 & 153 & 690 \\
\hline Raw hides, leather, and articles thereof & 12 & 7 & 21 & 25 \\
\hline Wood, cork, and articles thereof & 9 & 3 & 0 & 3 \\
\hline Wood pulp, paper, and paperboard & 110 & 113 & 146 & 206 \\
\hline Textiles and textile articles & 942 & 1,005 & 1,160 & 750 \\
\hline Footwear and other accessories & 3 & 3 & 3 & 6 \\
\hline Stone, plaster, cement, ceramic, and glassware & 175 & 162 & 217 & 452 \\
\hline Pearls, precious stones, and precious metals & 237 & 276 & 224 & 120 \\
\hline Base metals and related products & 2,656 & 3,052 & 2,997 & 3,279 \\
\hline Machinery and electrical equipment & 163 & 246 & 95 & 130 \\
\hline Vehicles and other transport equipment & 158 & 135 & 286 & 36 \\
\hline Optical and medical equipment & 1 & 2 & 1 & 8 \\
\hline Arms and ammunition & $\ldots$ & $\ldots$ & $\ldots$ & $\ldots$ \\
\hline Miscellaneous manufactured goods & 30 & 41 & 26 & 89 \\
\hline Works of art and antiques & 12 & 20 & 8 & 4 \\
\hline Total exports $2 /$ & 5,934 & 6,561 & 7,012 & 7,491 \\
\hline
\end{tabular}

Source: Central Bank of the United Arab Emirates.

1/ Exports of the Emirates of Abu Dhabi, Dubai, and Sharjah. Pre-1999 data not available.

2/ Data exclude free zone exports and "re-exports." 
Table 40. United Arab Emirates: Direction of Trade: Imports, 1998-2003 1/

(In percent of total)

\begin{tabular}{|c|c|c|c|c|c|c|}
\hline & 1998 & 1999 & 2000 & 2001 & 2002 & $\frac{\text { Prel. }}{2003}$ \\
\hline Total imports, c.i.f. & 100.0 & 100.0 & 100.0 & 100.0 & 100.0 & 100.0 \\
\hline Industrial countries & 52.2 & 52.4 & 52.7 & 56.8 & 57.8 & 58.7 \\
\hline \multicolumn{7}{|l|}{ Of which } \\
\hline Japan & 9.9 & 10.1 & 10.3 & 7.9 & 8.7 & 7.3 \\
\hline United States & 9.3 & 9.5 & 9.6 & 7.6 & 7.7 & 7.9 \\
\hline United Kingdom & 8.8 & 8.6 & 8.8 & 6.8 & 7.4 & 5.8 \\
\hline Italy & 5.6 & 5.9 & 6.1 & 4.7 & 5.1 & 5.0 \\
\hline Germany & 6.8 & 6.5 & 6.5 & 7.6 & 7.1 & 7.5 \\
\hline France & 3.8 & 3.9 & 4.0 & 6.0 & 6.6 & 7.8 \\
\hline Netherlands & 1.8 & 1.6 & 1.7 & 2.1 & 2.5 & 2.8 \\
\hline Australia & 1.9 & 1.8 & 1.7 & 2.1 & 2.1 & 2.6 \\
\hline Switzerland & 1.7 & 1.5 & 1.4 & 5.6 & 3.6 & 2.9 \\
\hline Developing countries & 46.8 & 46.5 & 46.3 & 43.2 & 42.2 & 41.3 \\
\hline Arab countries & 5.8 & 5.7 & 5.5 & 8.6 & 7.6 & 7.4 \\
\hline Of which: Saudi Arabia & 3.1 & 2.9 & 3.0 & 3.3 & 3.4 & 2.3 \\
\hline Other developing countries & 41.0 & 41.2 & 41.3 & 34.6 & 34.6 & 33.9 \\
\hline \multicolumn{7}{|l|}{ Of which } \\
\hline India & 5.9 & 6.1 & 6.8 & 6.2 & 6.7 & 4.7 \\
\hline Korea & 5.4 & 5.3 & 5.4 & 5.2 & 5.3 & 3.7 \\
\hline Hong Kong, SAR & 0.7 & 0.7 & 0.6 & 1.4 & 1.2 & 3.8 \\
\hline China & 7.9 & 8.2 & 8.4 & 7.5 & 8.2 & 11.3 \\
\hline Pakistan & 1.2 & 1.0 & 1.0 & 1.0 & 1.1 & 1.2 \\
\hline Iran & 1.5 & 1.4 & 1.3 & 1.2 & 1.2 & 0.8 \\
\hline Other unspecified & 1.0 & 1.1 & 1.2 & 1.0 & 0.0 & 0.0 \\
\hline
\end{tabular}

Source: IMF, Direction of Trade Statistics .

1/ Based on partner country information. 
Table 41. United Arab Emirates: Direction of Trade: Exports, 1998-2003 1/

(In percent of total)

\begin{tabular}{|c|c|c|c|c|c|c|}
\hline & 1998 & 1999 & 2000 & 2001 & 2002 & $\frac{\text { Prel. }}{2003}$ \\
\hline Total exports & 100.0 & 100.0 & 100.0 & 100.0 & 100.0 & 100.0 \\
\hline Industrial countries & 39.7 & 45.8 & 46.1 & 39.8 & 37.1 & 39.5 \\
\hline \multicolumn{7}{|l|}{ Of which } \\
\hline Japan & 29.7 & 36.5 & 36.1 & 29.5 & 27.2 & 28.0 \\
\hline United States & 6.4 & 2.2 & 2.4 & 2.9 & 2.3 & 2.4 \\
\hline United Kingdom & 3.3 & 1.8 & 1.8 & 2.1 & 2.7 & 2.7 \\
\hline Australia & 0.8 & 1.9 & 1.8 & 1.1 & 0.5 & 1.1 \\
\hline France & 0.4 & 0.2 & 0.3 & 1.3 & 1.1 & 1.1 \\
\hline Germany & 0.7 & 0.7 & 0.9 & 0.4 & 0.4 & 0.5 \\
\hline Italy & 0.4 & 0.6 & 0.7 & 0.3 & 0.3 & 0.4 \\
\hline Netherlands & 0.3 & 0.2 & 0.2 & 0.3 & 0.3 & 0.5 \\
\hline Developing countries & 42.9 & 42.3 & 42.5 & 45.3 & 46.8 & 45.5 \\
\hline Arab countries & 9.9 & 10.2 & 10.4 & 8.1 & 8.4 & 7.8 \\
\hline \multicolumn{7}{|l|}{ Of which } \\
\hline Oman & 5.1 & 3.6 & 3.1 & 3.8 & 3.7 & 3.3 \\
\hline Saudi Arabia & 1.8 & 1.4 & 1.7 & 1.3 & 1.3 & 1.2 \\
\hline Other developing countries & 33.0 & 32.1 & 32.8 & 37.2 & 38.4 & 37.8 \\
\hline \multicolumn{7}{|l|}{ Of which } \\
\hline Korea & 7.6 & 7.2 & 7.0 & 10.6 & 9.9 & 9.2 \\
\hline Iran & 2.0 & 3.5 & 3.7 & 3.4 & 4.3 & 3.9 \\
\hline Singapore & 3.8 & 6.4 & 6.1 & 3.1 & 3.7 & 3.7 \\
\hline India & 6.4 & 7.2 & 7.4 & 2.7 & 2.9 & 2.7 \\
\hline Thailand & 2.6 & 2.4 & 2.1 & 3.5 & 3.3 & 3.9 \\
\hline Kenya & 1.1 & 1.1 & 1.3 & 0.0 & 1.0 & 0.9 \\
\hline Hong Kong, SAR & 0.9 & 1.2 & 1.1 & 1.6 & 2.2 & 2.3 \\
\hline Pakistan & 2.1 & 2.0 & 2.4 & 3.1 & 3.1 & 3.2 \\
\hline Philippines & 0.9 & 0.9 & 0.8 & 1.4 & 0.9 & 1.0 \\
\hline Other unspecified & 17.4 & 11.9 & 11.4 & 14.8 & 16.2 & 15.0 \\
\hline
\end{tabular}

Source: IMF, Direction of Trade Statistics.

1/ Based on partner country information. 